\title{
Arabinose-derived ketones as Catalysts for Asymmetric Epoxidation of alkenes
}

\section{Supporting Information}

Tony K. M. Shing, ${ }^{*}$ Gulice Y. C. Leung and To Luk

General

General Benzoylation Procedure of Benzyl Epoxide

General Desilylation Procedure

General Procedure for preparing corresponding Acetate after Desilylation

General Detritylation Procedure

NMR spectral and HPLC data for the determination of the enantiomeric excess

of the epoxides

Spectral characterization data for new compounds

${ }^{1} \mathrm{H}$ : and ${ }^{13} \mathrm{C} \quad 4$

${ }^{1} \mathrm{H}$ : and ${ }^{13} \mathrm{C} \quad 7$

S25-26

${ }^{1} \mathrm{H}$ : and ${ }^{13} \mathrm{C} \quad 8$

S27-28

${ }^{1} \mathrm{H}$ : and ${ }^{13} \mathrm{C} \quad 9$

S29-30

${ }^{1} \mathrm{H}$ : and ${ }^{13} \mathrm{C} \quad \mathbf{1 0}$

S31-32

${ }^{1} \mathrm{H}$ : and ${ }^{13} \mathrm{C} \quad \mathbf{1 1}$

S33-34

${ }^{1} \mathrm{H}$ : and ${ }^{13} \mathrm{C} \quad \mathbf{1 2}$

S35-36

${ }^{1} \mathrm{H}$ : and ${ }^{13} \mathrm{C} \quad \mathbf{1 3}$

S37-38

${ }^{1} \mathrm{H}$ : and ${ }^{13} \mathrm{C} \quad \mathbf{1 4}$

S39-40

${ }^{1} \mathrm{H}$ : and ${ }^{13} \mathrm{C} \quad \mathbf{1 5}$

S41-42

${ }^{1} \mathrm{H}$ : and ${ }^{13} \mathrm{C} \quad \mathbf{1 6}$

S43-44

${ }^{1} \mathrm{H}$ : and ${ }^{13} \mathrm{C} \quad \mathbf{1 7}$

S45-46

${ }^{1} \mathrm{H}$ : and ${ }^{13} \mathrm{C} \quad \mathbf{1 8}$

S47-48

${ }^{1} \mathrm{H}$ : and ${ }^{13} \mathrm{C} \quad 19$

S49-50

${ }^{1} \mathrm{H}$ : and ${ }^{13} \mathrm{C} \quad \mathbf{2 0}$

S51-52 
General Melting points were reported in Celsius degrees and are uncorrected. Optical rotations were measured at $589 \mathrm{~nm}$. Infrared (IR) spectra were recorded on FT-IR spectrophotometer as thin films on $\mathrm{KBr}$ discs. Nuclear magnetic resonance (NMR) spectra were measured at $300.13 \mathrm{MHz}\left({ }^{1} \mathrm{H}\right)$ or at $75.47 \mathrm{MHz}\left({ }^{13} \mathrm{C}\right)$ in $\mathrm{CDCl}_{3}$ solutions, unless stated otherwise. All chemical shifts were recorded in ppm relative to tetramethylsilane $(\delta=0.0)$. Spin-spin coupling constants $(J)$ in Hz were measured directly from the spectra. All reactions were monitored by analytical thin-layer chromatography (TLC) on aluminum-precoated plates of silica gel $60 \mathrm{~F}_{254}$ and compounds were visualized with a spray of $5 \% \mathrm{w} / \mathrm{v}$ dodecamolybdophosphoric acid in $\mathrm{EtOH}$ and subsequent heating. silica gel 60 (230-400 mesh) was used for all flash column chromatography. The reaction $p \mathrm{H}$ was monitored by a $p \mathrm{H}$ meter with a $p \mathrm{H}$ triode electrode. All solvents were reagent grade unless otherwise stated. Toluene, benzene and THF were fleshly distilled from $\mathrm{Na} /$ benzophenone ketyl under nitrogen. $\mathrm{CH}_{2} \mathrm{Cl}_{2}$ was freshly distilled from $\mathrm{CaH}_{2}$ under $\mathrm{N}_{2}$. Other reagents were purchased from commercial suppliers and used without purification. All hexanes used are n-hexane. 


\section{General Benzoylation Procedure of Benzyl Epoxide:}

To a solution of 1-benzyloxy-3,4-epoxyhexane (Table 3, Entry 2) (37 mg, $0.18 \mathrm{mmol}$ ) in $7 \mathrm{ml}$ of $\mathrm{CCl}_{4} / \mathrm{CH}_{3} \mathrm{CN} / \mathrm{H}_{2} \mathrm{O}(2: 2: 3 \mathrm{v} / \mathrm{v} / \mathrm{v})$ were added a catalytic amount of ruthenium (IV) oxide (ca. $1 \mathrm{mg}$ ) and sodium periodate $(150 \mathrm{mg}, 0.7 \mathrm{mmol}$ ) at room temperature under $\mathrm{N}_{2}$. Upon stirring under $\mathrm{N}_{2}$ for $2 \mathrm{~h}$, the reaction mixture was quenched by saturated aq. $\mathrm{Na}_{2} \mathrm{~S}_{2} \mathrm{O}_{3}$ solution $(3 \mathrm{ml})$, extracted with diethyl ether (10 $\mathrm{mL}$ ), washed with water, dried with anhydrous $\mathrm{MgSO}_{4}$ and filtered. The filtrate was concentrated under reduced pressure. The crude residue was purified by flash column chromatography to afford 1-benzoyloxy-3,4-epoxyhexane as a colorless oil (38 mg, 96\%): $R_{f} 0.33$ (hexane-Et ${ }_{2} \mathrm{O}, 3: 1$ ).

\section{General Desilylation Procedure:}

To a solution of 1-tert-butyldimethylsilyloxy-3,4-epoxyhexane (Table 3, Entry 3) (50 $\mathrm{mg}, 0.22 \mathrm{mmol})$ in THF (2 mL) was added dropwise tetrabutylammonium fluoride (1 $\mathrm{M}$ in THF, $0.5 \mathrm{~mL}, 0.5 \mathrm{mmol}$ ) under $\mathrm{N}_{2}$. Upon stirring under $\mathrm{N}_{2}$ for $3 \mathrm{~h}$, the reaction mixture was diluted with diethyl ether $(10 \mathrm{~mL})$, washed with water, extracted with diethyl ether $(10 \mathrm{~mL})$, dried with anhydrous $\mathrm{MgSO}_{4}$ and filtered. The filtrate was concentrated under reduced pressure. The crude residue was purified by flash column chromatography to afford trans-3-hexen-1-ol oxide as a colorless oil (21 mg, 83\%): $R_{f}$ 0.2 (hexane- $\mathrm{Et}_{2} \mathrm{O}, 1: 2$ ). 


\section{General Procedure for Preparing the Corresponding Acetate after Desilylation:}

To a solution of trans-3-hexen-1-ol oxide $(21 \mathrm{mg}, 0.18 \mathrm{mmol})$ in dry $\mathrm{CH}_{2} \mathrm{Cl}_{2}(3 \mathrm{~mL})$ were added triethylamine $(0.14 \mathrm{~mL}, 1.08 \mathrm{mmol})$ and acetic anhydride $(0.05 \mathrm{~mL}, 0.54$ mmol) at room temperature. The reaction mixture was stirred for $3 \mathrm{~h}$. The mixture was treated with saturated aq. $\mathrm{NaHCO}_{3}$ and extracted with $\mathrm{Et}_{2} \mathrm{O}(3 \times 5 \mathrm{~mL})$. The combined organic extracts were dried with anhydrous $\mathrm{MgSO}_{4}$ and filtered. The filtrate was concentrated under reduced pressure. The crude residue was purified by flash column chromatography to afford the corresponding acetate $(25 \mathrm{mg}, 88 \%): R_{f}$ 0.2 (hexane- $\mathrm{Et}_{2} \mathrm{O}, 3: 1$ ).

\section{General Detritylation Procedure:}

To a solution of 1-trityloxy-3,4-epoxyhexane (Table 3, Entry 4) (40 mg, $0.11 \mathrm{mmol}$ ) in $10 \mathrm{ml}$ ethanol/ethyl acetate $(9: 1 \mathrm{v} / \mathrm{v})$ was added $10 \%$ palladium on charcoal $(0.1 \mathrm{~g})$. The mixture was hydrogenated/hydrogen balloon at room temperature for $72 \mathrm{~h}$. The mixture was filtered and the filtrate was concentrated under reduced pressure. The crude residue was purified by flash column chromatography to afford trans-3-hexen-1-ol oxide as a colorless oil (9 mg, 71\%): $R_{f} 0.2$ (hexane-Et ${ }_{2} \mathrm{O}, 1: 2$ ). 


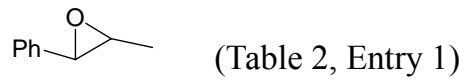

${ }^{1}$ H NMR Conditions: $(6 \mathrm{mg})$ was dissolved in $\mathrm{CDCl}_{3}(0.6 \mathrm{~mL})$, ratio of epoxide $\left.: \mathrm{Eu}(\mathrm{hfc})_{3} \approx 10: 6.8(\mathrm{w} / \mathrm{w})\right\}$

ee measured $\mathrm{Ph} \stackrel{\mathrm{D}}{\mathrm{C}}$

Recemic Epoxide
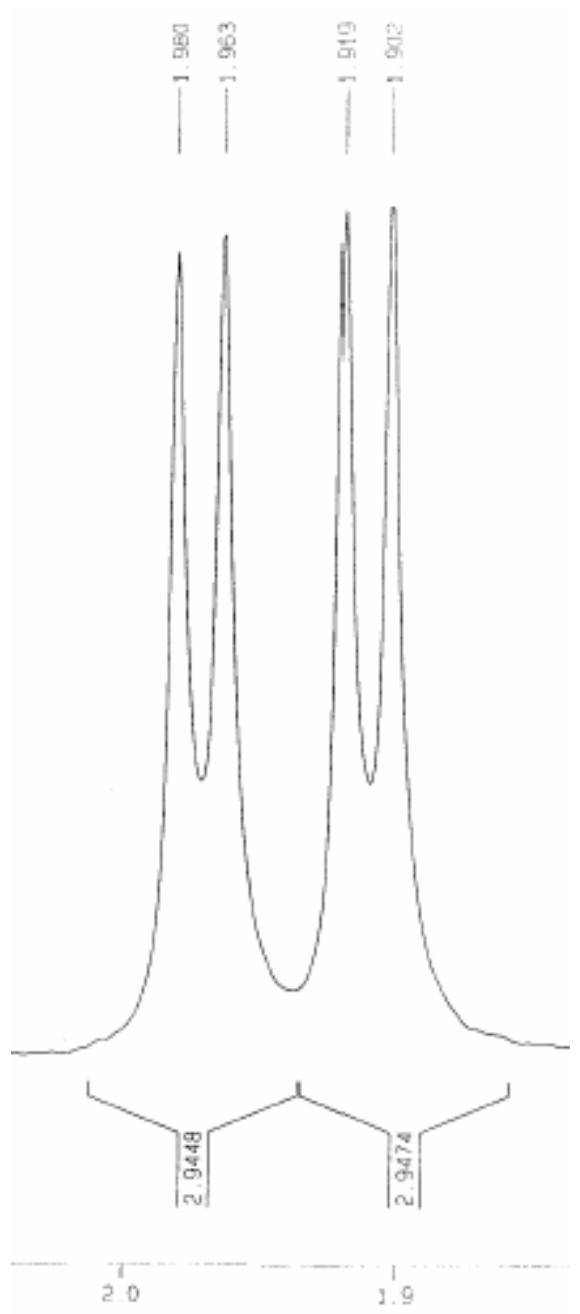

Chiral Epoxide

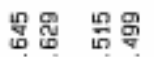

$\tilde{i} \tilde{i}$

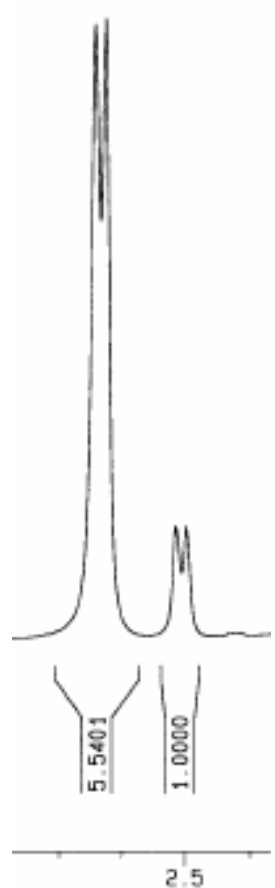


$\mathrm{Ph} \stackrel{\text { ic }}{\mathrm{Ph}}$ (Table 2, Entry 8)

HPLC Conditions: Column: Chiralcel OD-H.

Eluent: Hexane/2-propanol (90/10); Flow rate: $0.5 \mathrm{ml} / \mathrm{min}$;

Detection:UV 254nm

\section{Racemic Epoxide}

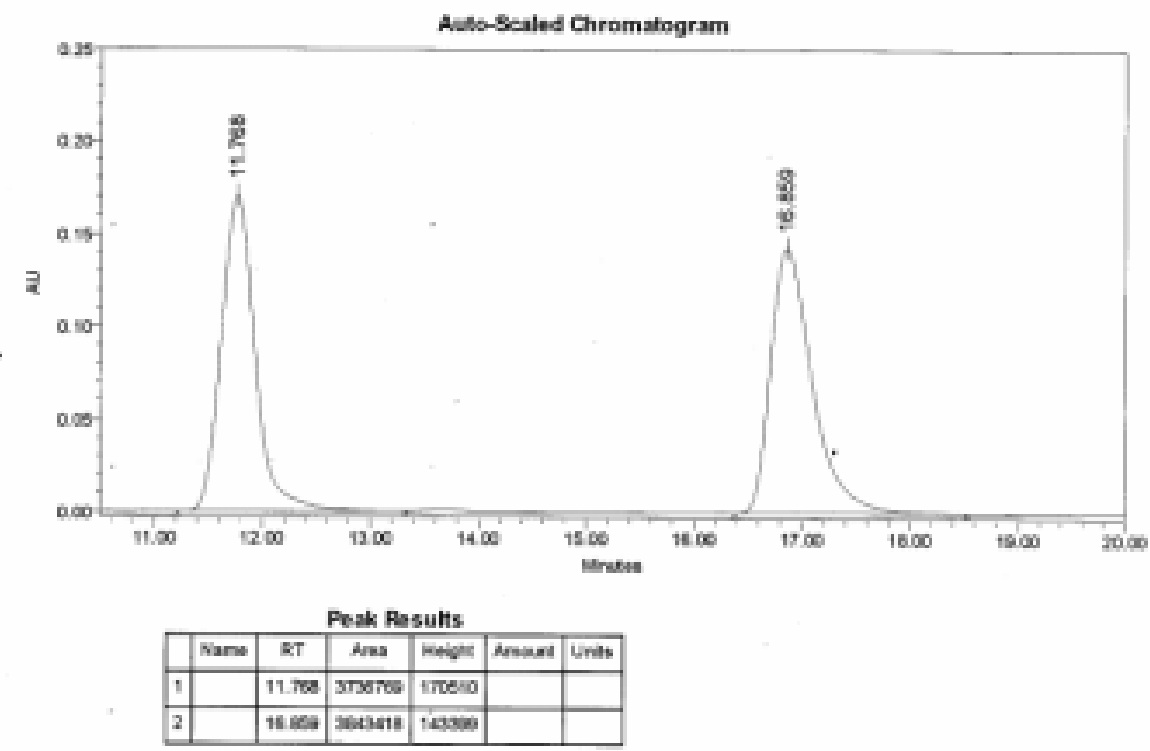

\section{Chiral Epoxide}

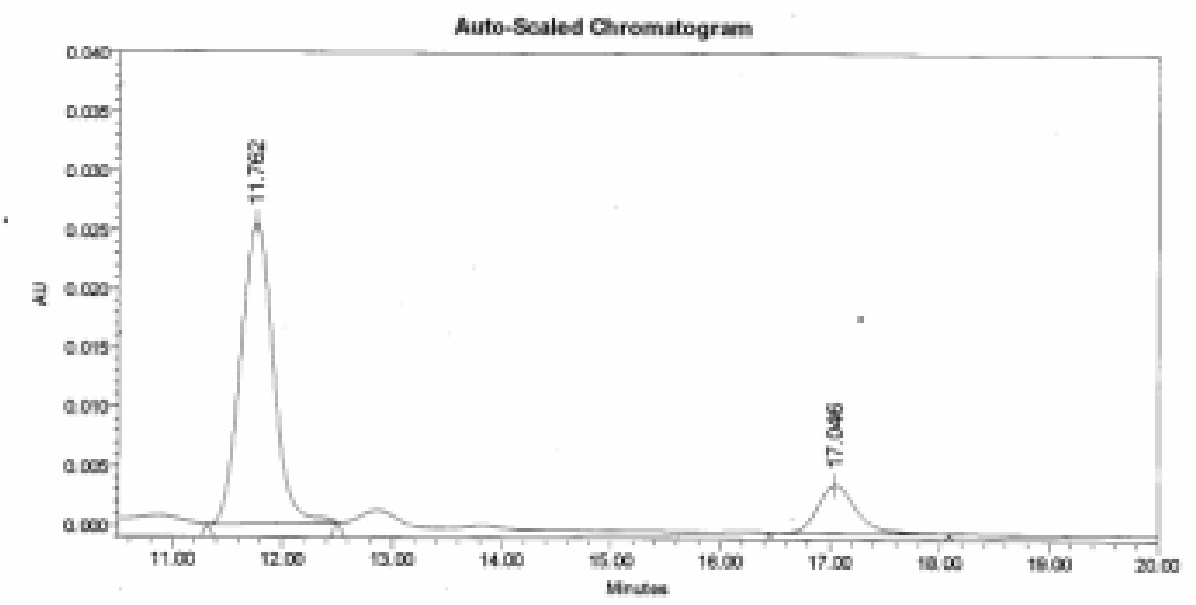

\begin{tabular}{|c|c|c|c|c|c|c|}
\hline \multicolumn{7}{|c|}{ Peak Results } \\
\hline & Marse & $R T$ & Area & Hevor & Ameval & unts \\
\hline 1 & & $11 . \pi 00$ & 500251 & 29501 & & \\
\hline 2 & & 17048 & 2017es & 4286 & & \\
\hline
\end{tabular}




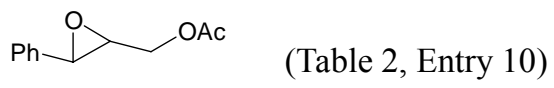

${ }^{1}$ H NMR Conditions: (5mg) was dissolved in $\mathrm{CDCl}_{3}(0.6 \mathrm{~mL})$,

ratio of epoxide $\left.: \mathrm{Eu}(\mathrm{hfc})_{3} \approx 10: 6.8(\mathrm{w} / \mathrm{w})\right\}$

ee measured $\mathrm{Ph} \stackrel{\mathrm{O}}{\mathrm{C} A \mathrm{C}}$

Recemic Epoxide
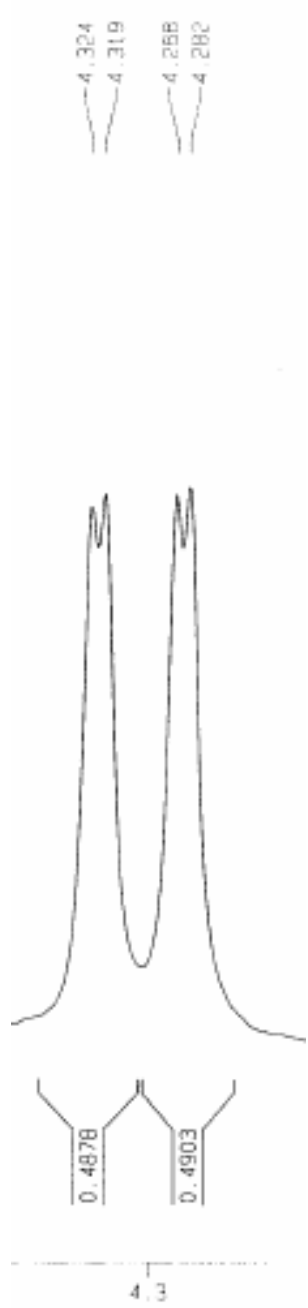

Chiral Epoxide

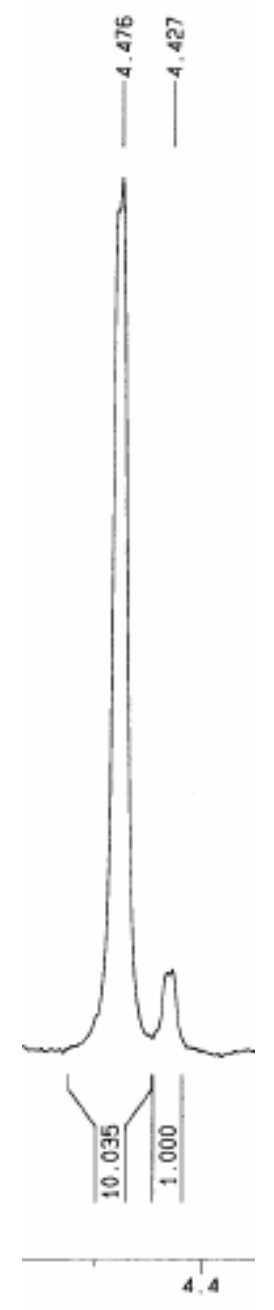

The absolute configuration was determined by comparing its free alcohol

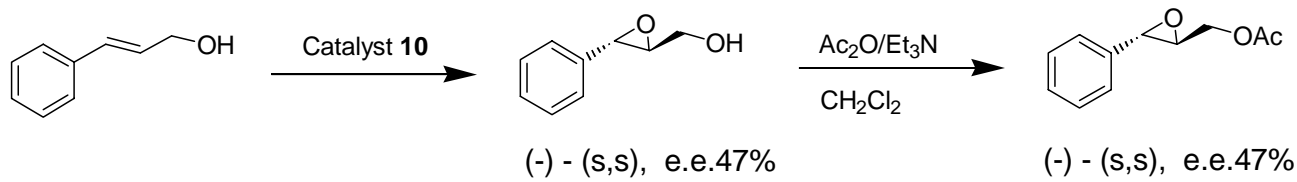


<smiles>[SeH3]OCC1OC1c1ccccc1</smiles>

(Table 2, Entry 16)

Enantioselectivity was determined after desilylation with TBAF

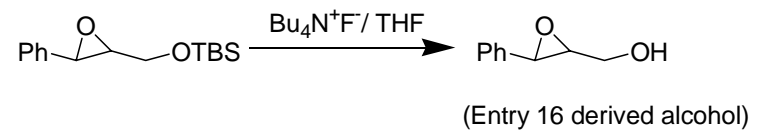

HPLC Conditions: Column: Chiralcel OD-H.

Eluent: Hexane/2-propanol (90/10); Flow rate: $0.5 \mathrm{ml} / \mathrm{min}$;

Detection:UV 254nm

\section{Racemic epoxide}

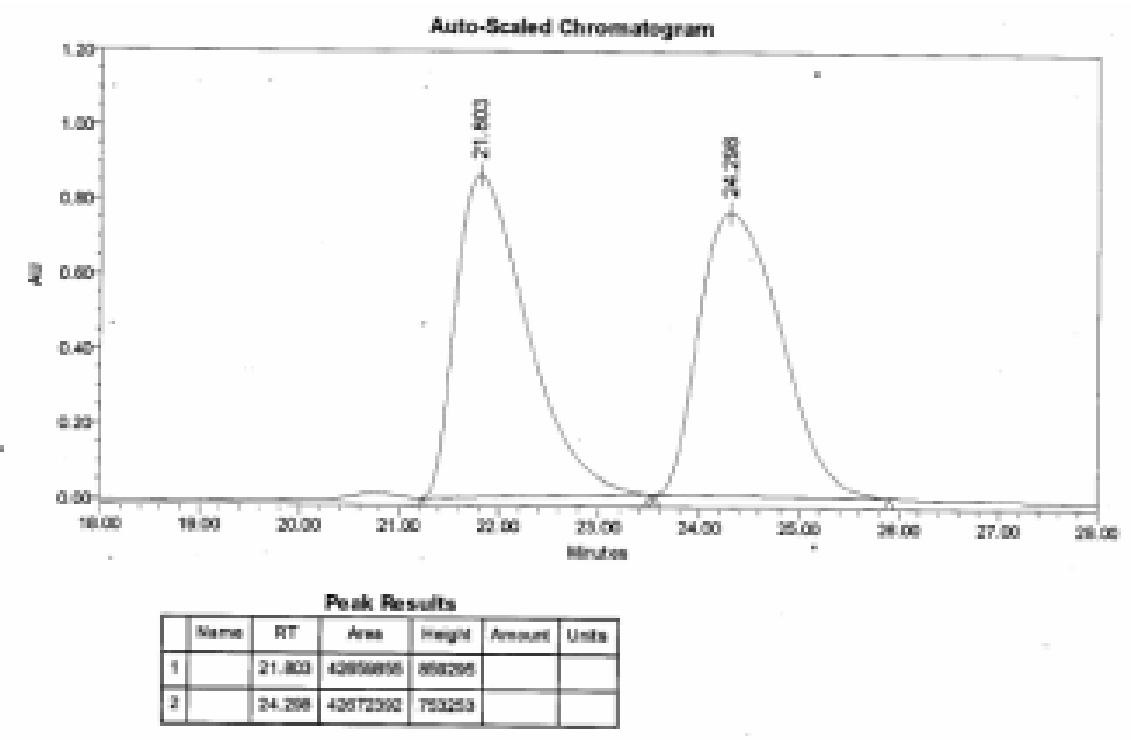

\section{Chiral Epoxide}

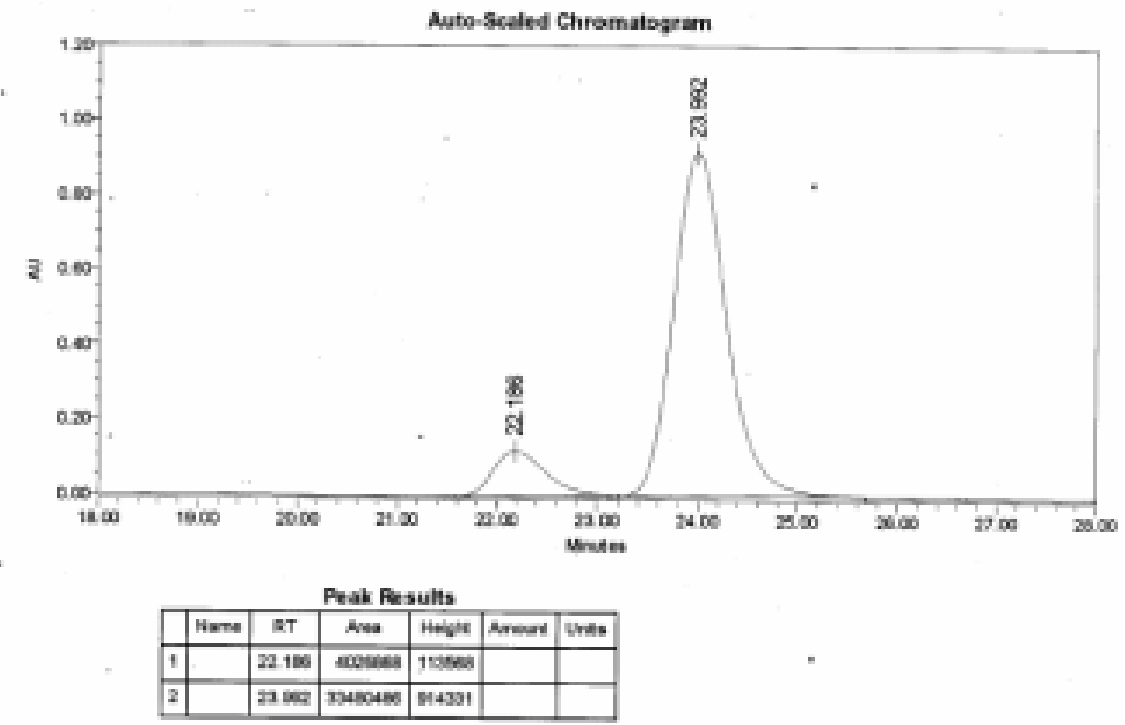




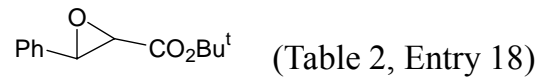

${ }^{1}$ H NMR Conditions: (5mg) was dissolved in $\mathrm{CDCl}_{3}(0.6 \mathrm{~mL})$,

ratio of epoxide $\left.: \mathrm{Eu}(\mathrm{hfc})_{3} \approx 10: 1.0(\mathrm{w} / \mathrm{w})\right\}$

ee measured $\mathrm{Ph} \stackrel{\mathrm{O}}{\mathrm{D}}>\mathrm{CO}_{2} \mathrm{Bu}^{\mathrm{t}}$

Racemic Epoxide


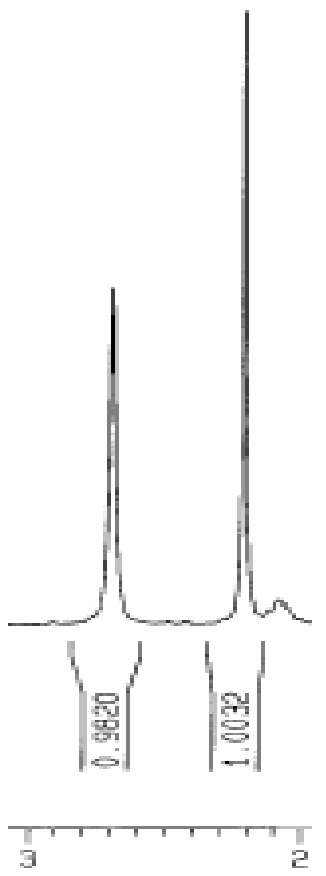

\section{Chiral Epoxide}

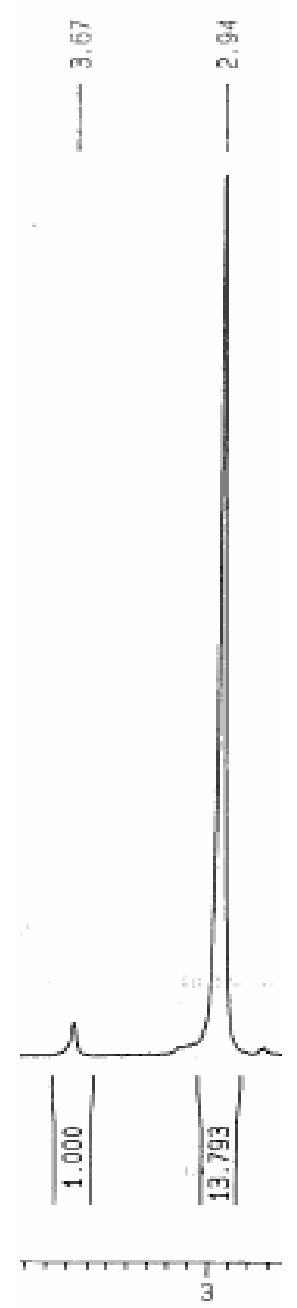




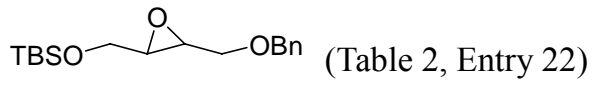

Enantioselectivity was determined after desilylation with TBAF and measured its acetate

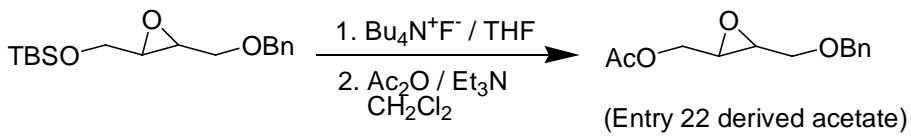

${ }^{1}$ H NMR Conditions: $\quad(4 \mathrm{mg})$ was dissolved in $\mathrm{C}_{6} \mathrm{D}_{6}(0.6 \mathrm{~mL})$, ratio of epoxide $\left.: \mathrm{Eu}(\mathrm{hfc})_{3} \approx 10: 6.3(\mathrm{w} / \mathrm{w})\right\}$

ee measured $A C D$ i $O B n$

Racemic epoxide

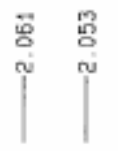

Chiral Epoxide

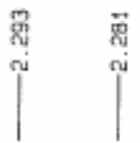

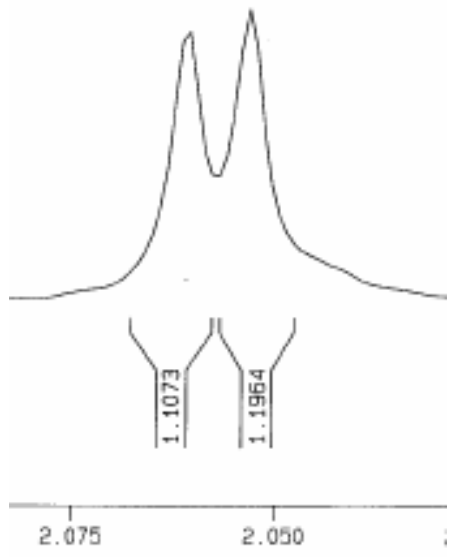

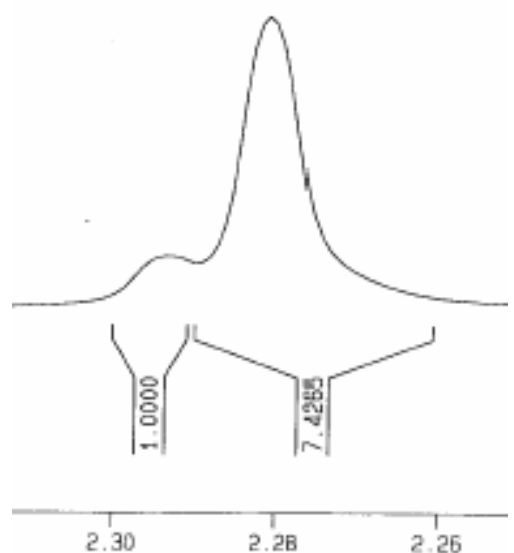




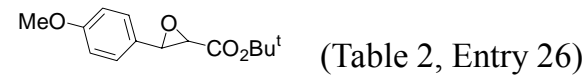

${ }^{1}$ H NMR Conditions: (5mg) was dissolved in $\mathrm{CDCl}_{3}(0.6 \mathrm{~mL})$,

ratio of epoxide $\left.: \mathrm{Eu}(\mathrm{hfc})_{3} \approx 10: 1.0(\mathrm{w} / \mathrm{w})\right\}$

ee measured $\mathrm{MeO}$ i

Racemic Epoxide

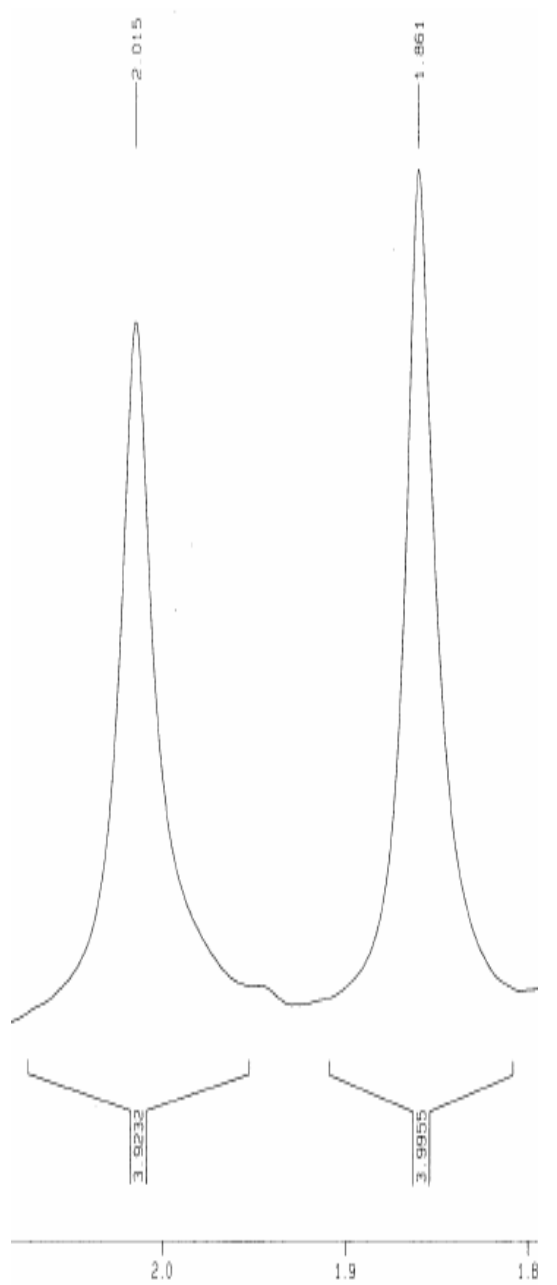

Chiral Epoxide
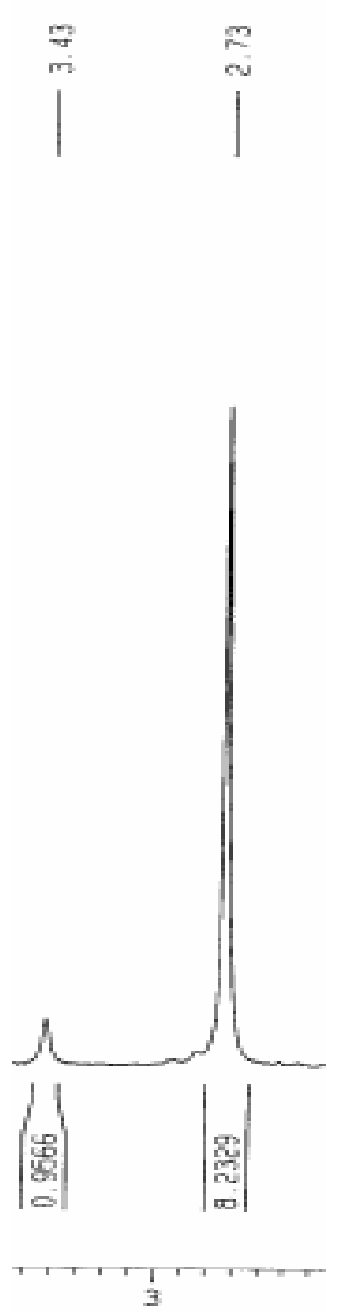
$\mathrm{MeO}$ i $\mathrm{CO}_{2} \mathrm{Me}$ (Table 2, Entry 30)

HPLC Conditions: Column: Chiralcel OD-H.

Eluent: Hexane/2-propanol (90/10); Flow rate: $1.0 \mathrm{ml} / \mathrm{min}$;

Detection:UV 220nm

Racemic epoxide

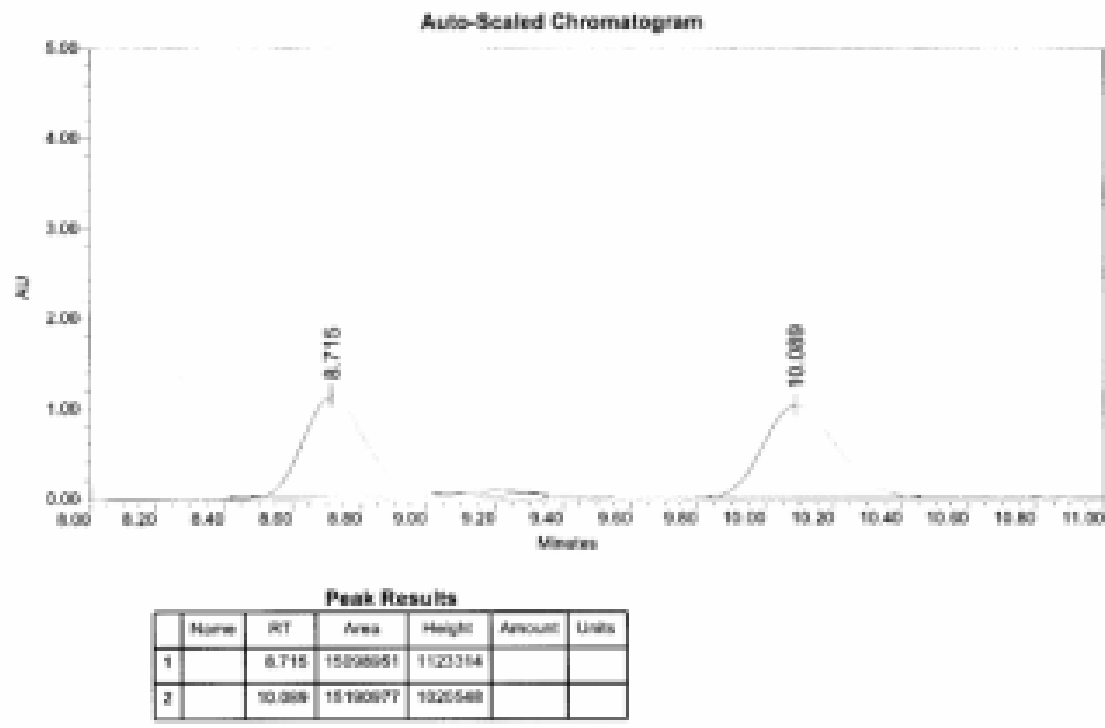

Chiral epoxide

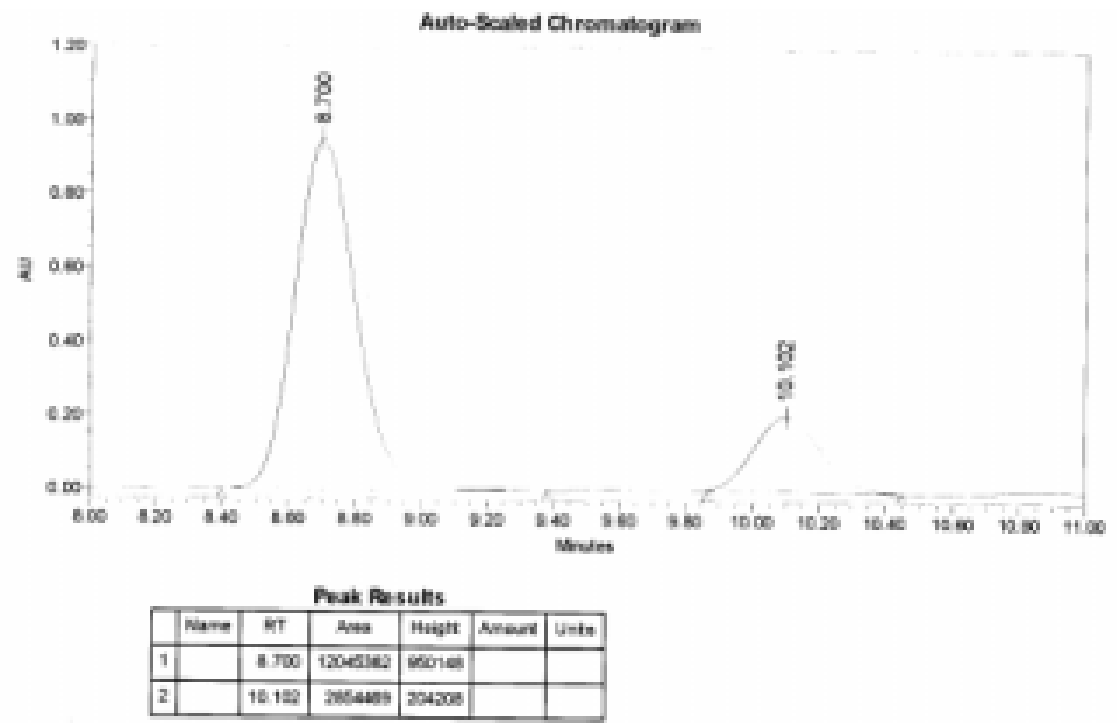


<smiles>CC1(c2ccccc2)OC1c1ccccc1</smiles>

(Table 2, Entry 36)

HPLC Conditions: Column: Chiralcel OD-H.

Eluent: Hexane/2-propanol (90/10); Flow rate: $0.5 \mathrm{ml} / \mathrm{min}$;

Detection:UV 254nm

\section{Racemic Epoxide}

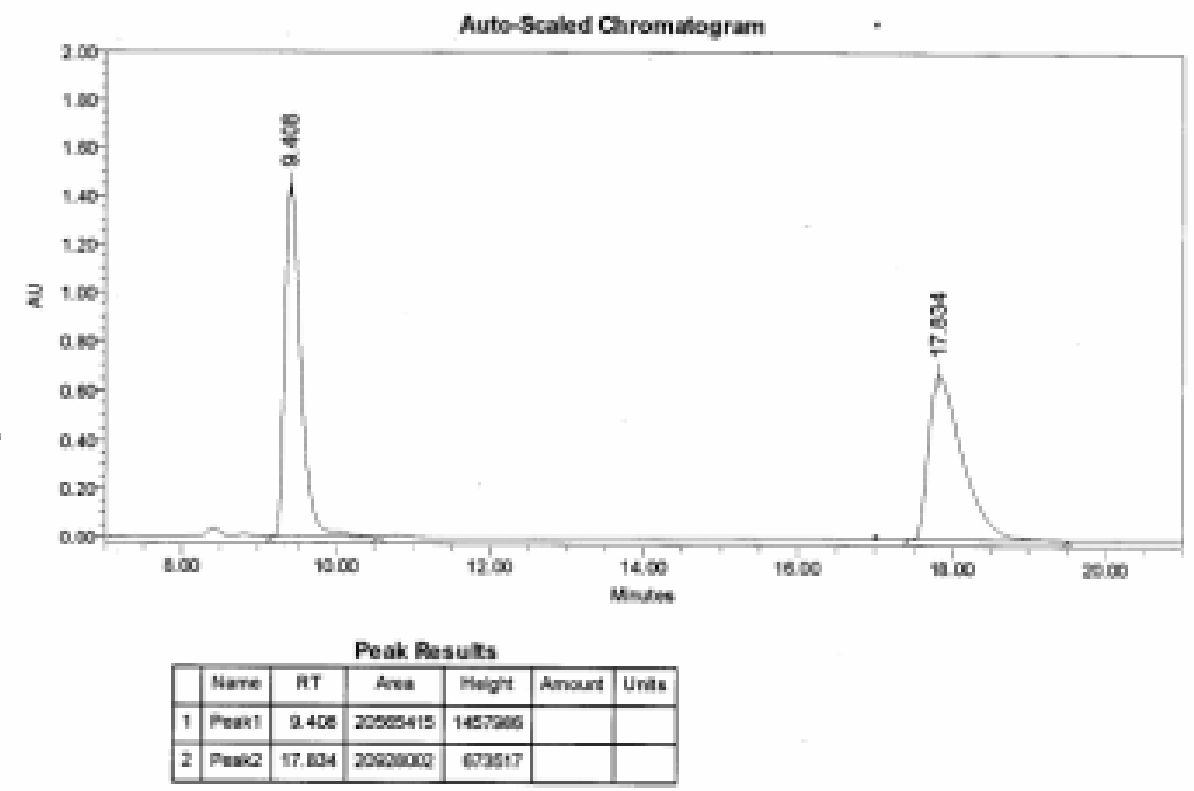

\section{Chiral Epoxide}

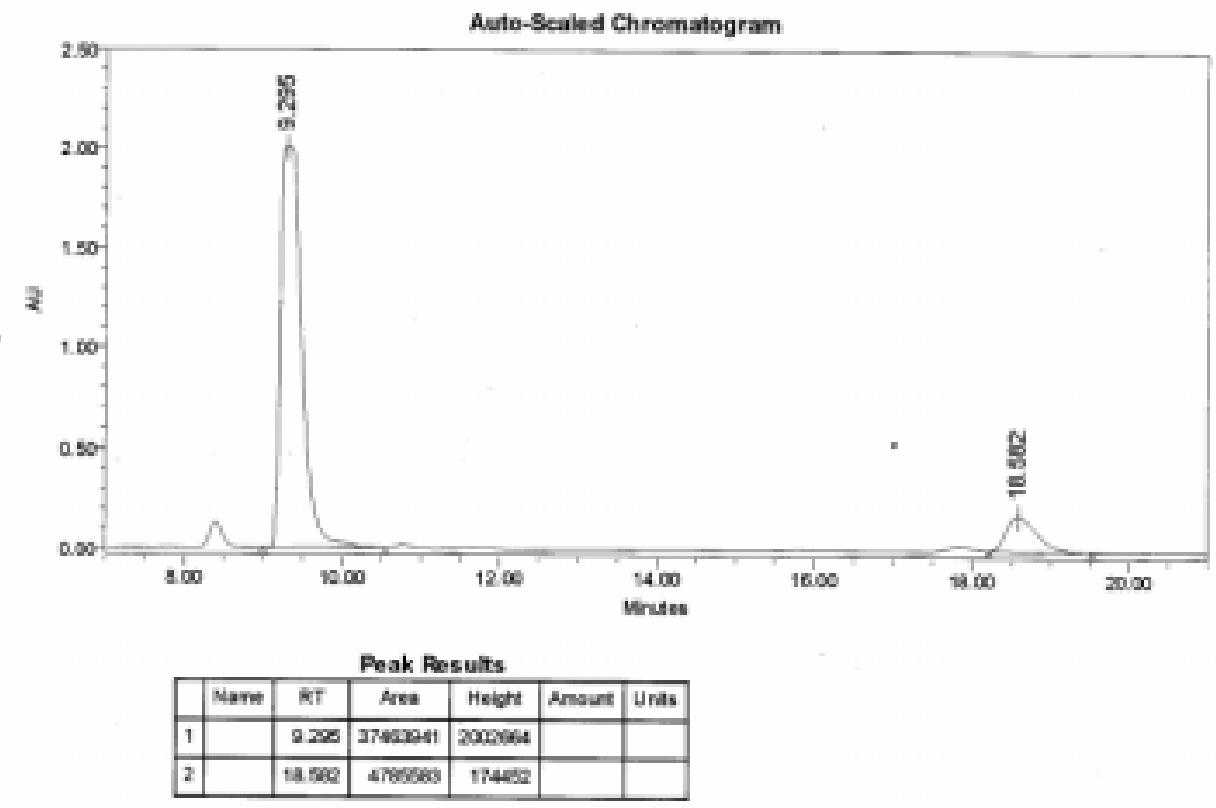




$$
\mathrm{Ph} \mathcal{O}_{\mathrm{Ph}}^{\mathrm{Ph}} \text { (Table 2, Entry 40) }
$$

HPLC Conditions: Column: Chiralcel OD-H.

Eluent: Hexane/2-propanol (90/10); Flow rate: $0.5 \mathrm{ml} / \mathrm{min}$;

Detection:UV 254nm

\section{Racemic Epoxide}

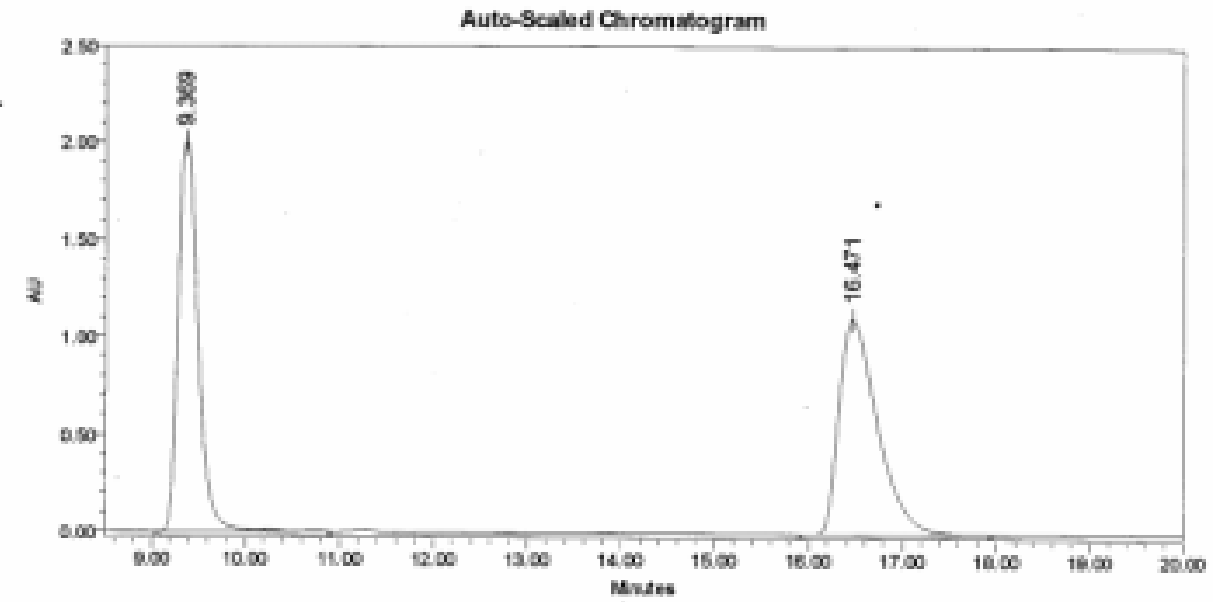

\begin{tabular}{|c|c|c|c|c|c|c|}
\hline \multicolumn{7}{|c|}{ Peak Results } \\
\hline & Name & RT & Avea & Meloht & Anourt & Unats \\
\hline 1 & & 2360 & 2047308 & 2004:-4 & & \\
\hline 2 & & 54 \& 1 & 3170008 & 1169715 & & \\
\hline
\end{tabular}

\section{Chiral Epoxide}

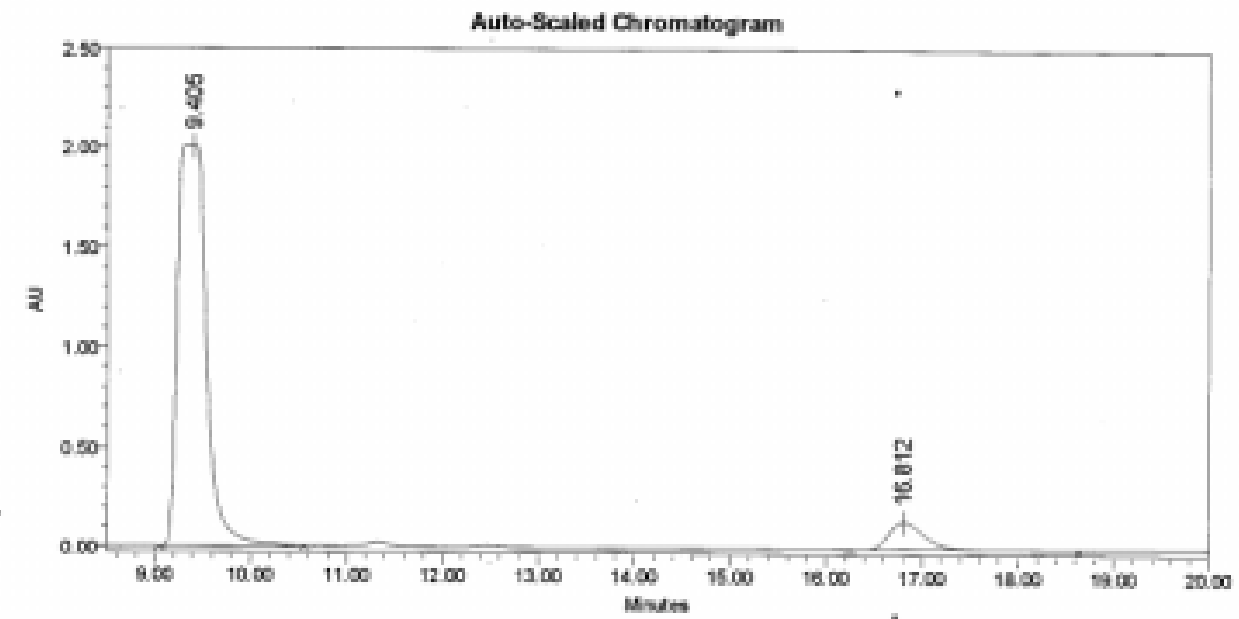

\begin{tabular}{|c|c|c|c|c|c|c|}
\hline \multicolumn{7}{|c|}{ Peak Bosults } \\
\hline & Narest & AT & Avos & Nelloht & Ameunt & Unta \\
\hline 1 & & 2.436 & 41711121 & 2004361 & & \\
\hline 2 & & 16.812 & 3532430 & 19eves & & \\
\hline
\end{tabular}


(Table 2, Entry 44)

${ }^{1}$ H NMR Conditions: (4 mg) was dissolved in $\mathrm{CDCl}_{3}(0.6 \mathrm{~mL})$, ratio of epoxide $\left.: \operatorname{Eu}(\mathrm{hfc})_{3} \approx 10: 6.3(\mathrm{w} / \mathrm{w})\right\}$

ee measured

Racemic epoxide

Chiral epoxide

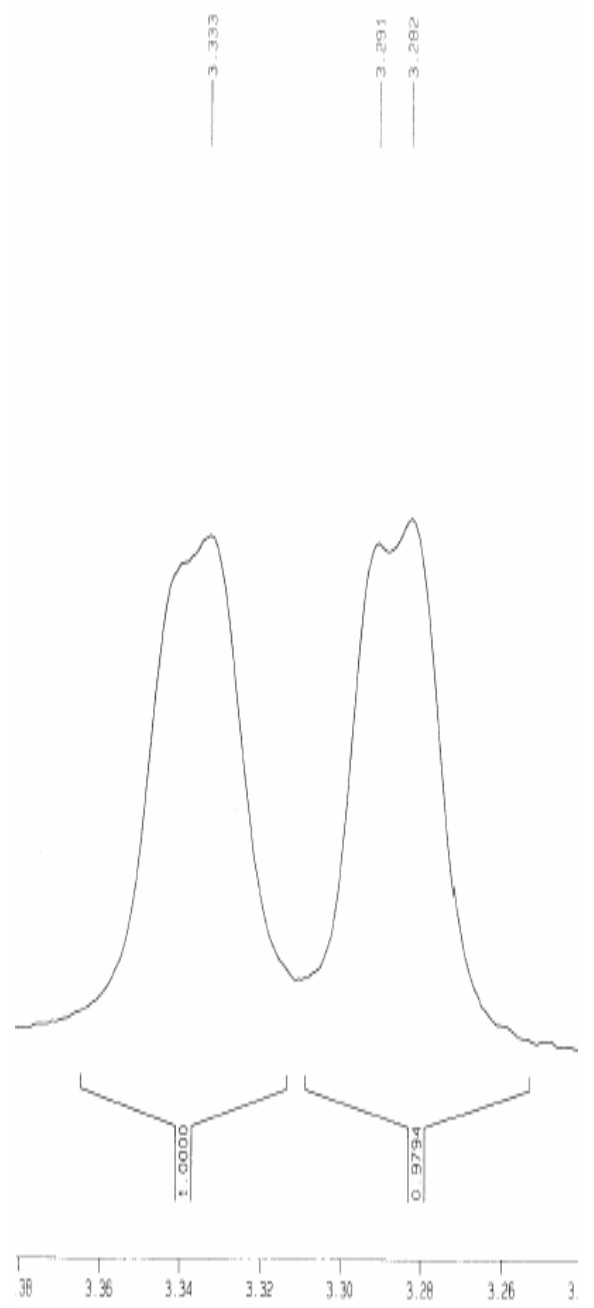

퐁

i
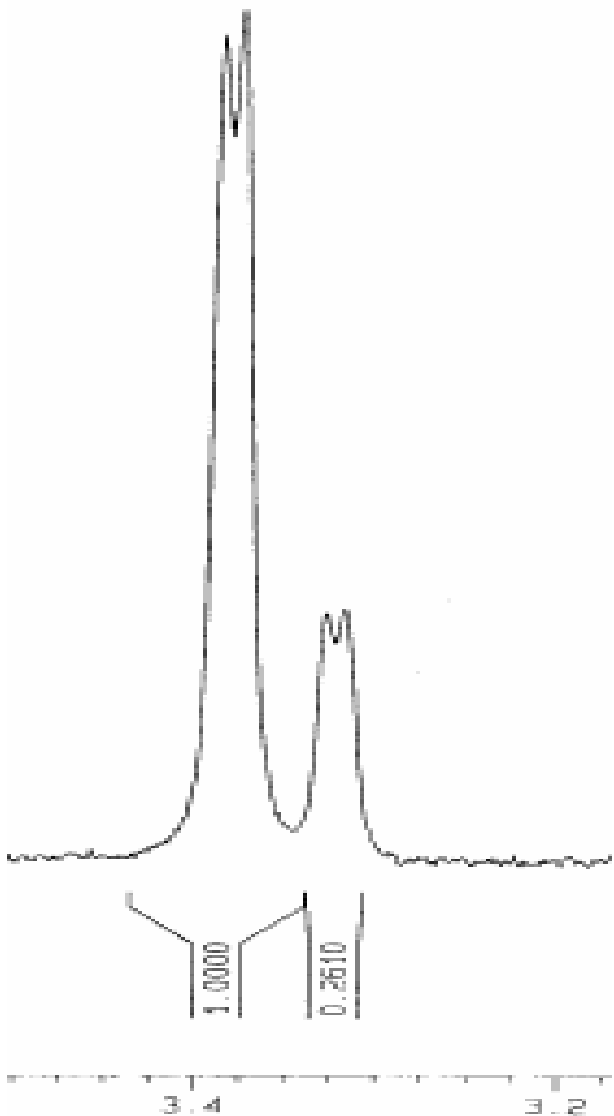


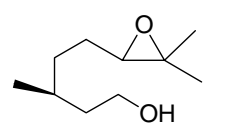

(Table 2, Entry 46)

Enantioselectivity was measured from its acetate

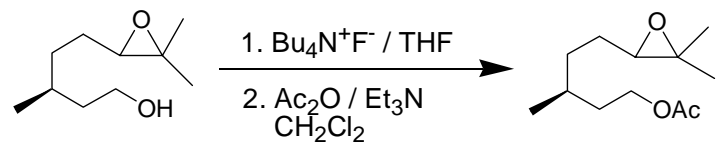

(Entry 46 derived acetate)

${ }^{1} \mathrm{H}$ NMR Conditions: $\quad(4 \mathrm{mg})$ was dissolved in $\mathrm{C}_{6} \mathrm{D}_{6}(0.6 \mathrm{~mL})$, ratio of epoxide $\left.: \mathrm{Eu}(\mathrm{hfc})_{3} \approx 10: 6.3(\mathrm{w} / \mathrm{w})\right\}$

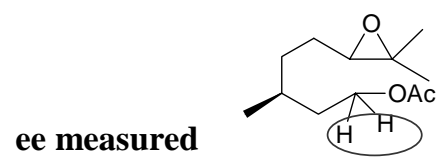

Racemic Epoxide

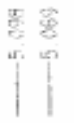

Chiral Epoixde

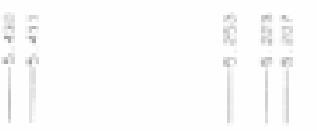

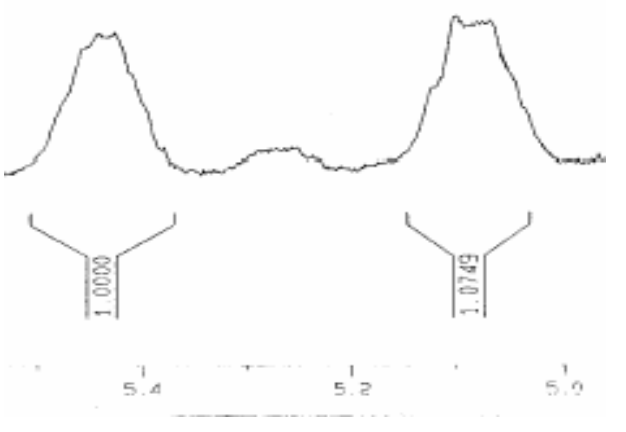




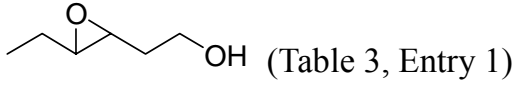

Enantioselectivity was measured from its acetate

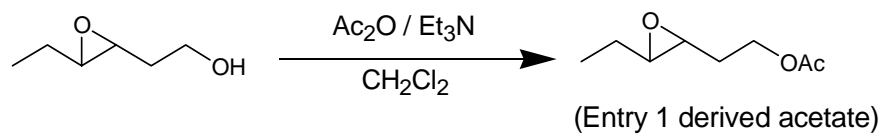

${ }^{1}$ H NMR Conditions: (5 mg) was dissolved in $\mathrm{C}_{6} \mathrm{D}_{6}(0.6 \mathrm{~mL})$,

ratio of epoxide $\left.: \operatorname{Eu}(\mathrm{hfc})_{3} \approx 10: 3.3(\mathrm{w} / \mathrm{w})\right\}$

ee measured<smiles>CCC1OC1CCCOc1ccccc1</smiles>

Racemic Epoxide

Chiral Epoxide
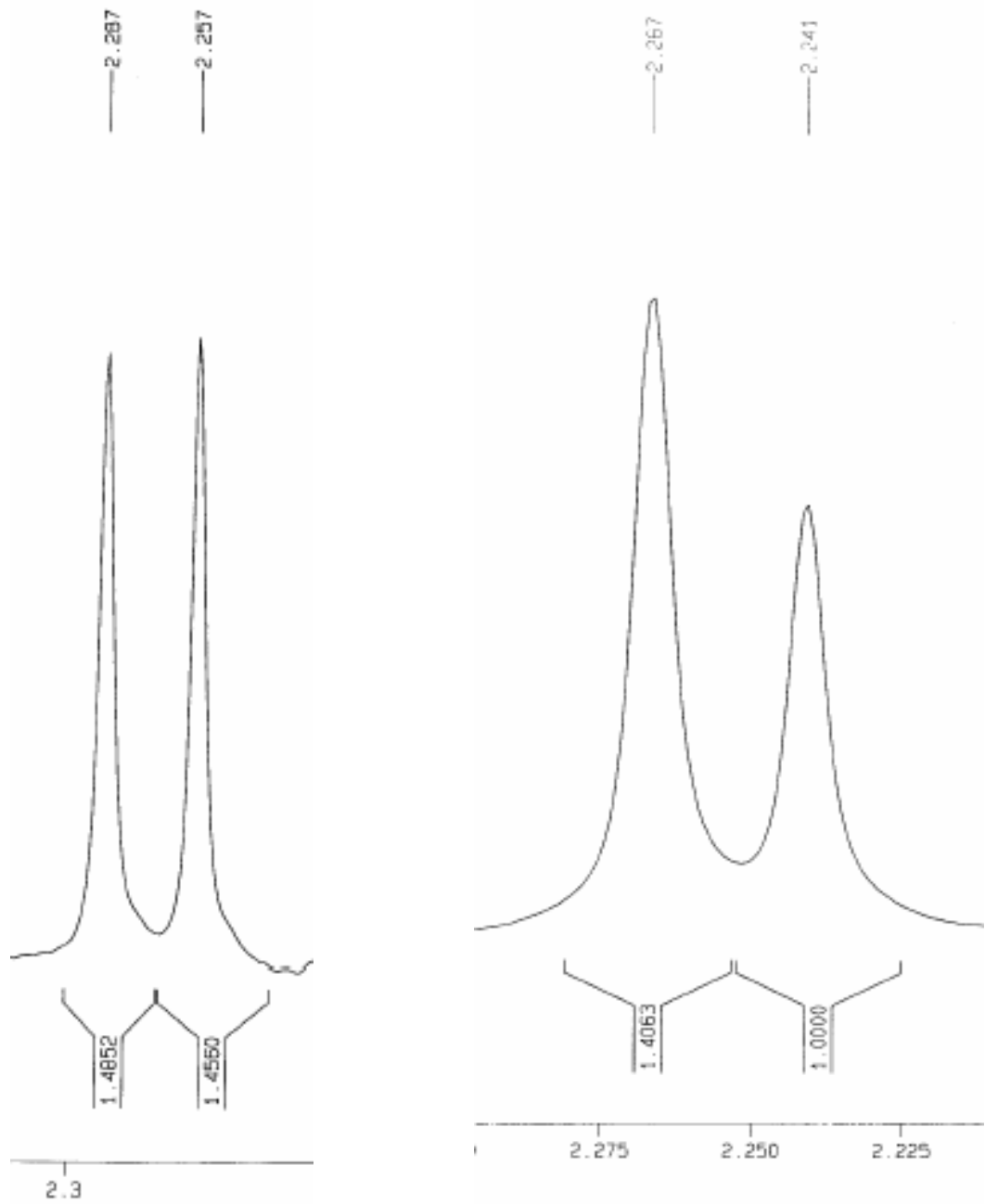

2.3 


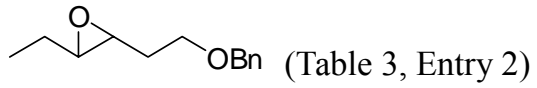

Enantioselectivity was measured from its benzoate

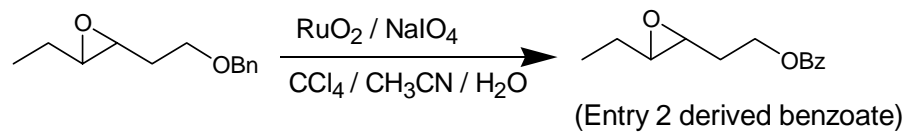

${ }^{1}$ H NMR Conditions: (5 mg) was dissolved in $\mathrm{CDCl}_{3}(0.6 \mathrm{~mL})$, ratio of epoxide : $\left.\mathrm{Eu}(\mathrm{hfc})_{3} \approx 10: 4.5(\mathrm{w} / \mathrm{w})\right\}$

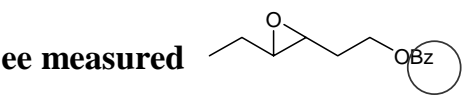

\section{Racemic Epoxide}
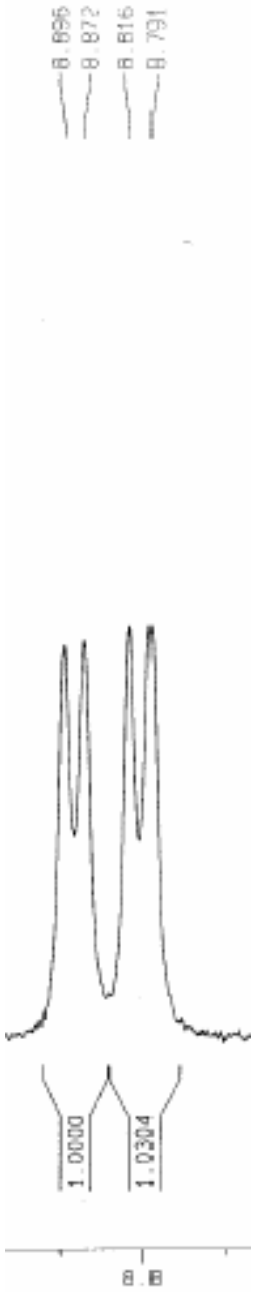

Chiral Epoxide
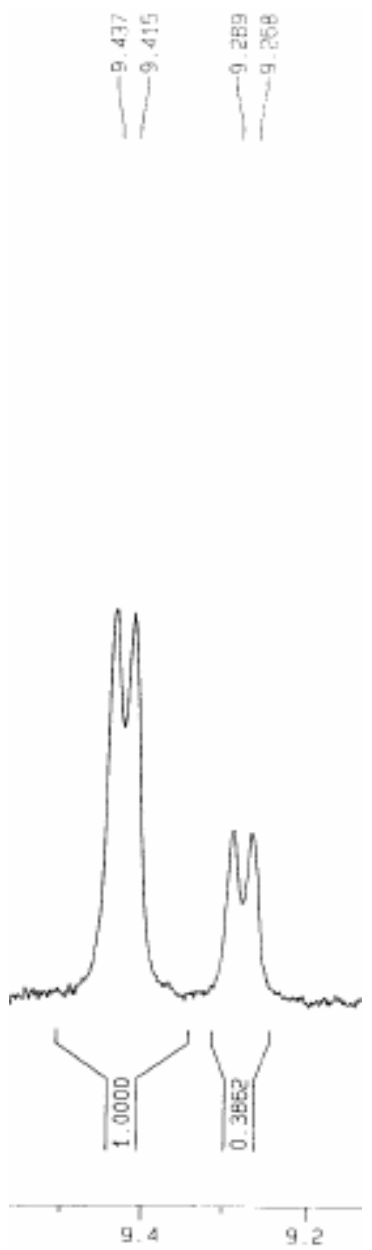


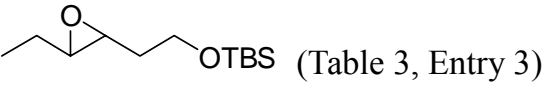

Enantioselectivity was determined after desilylation with TBAF and measured its acetate

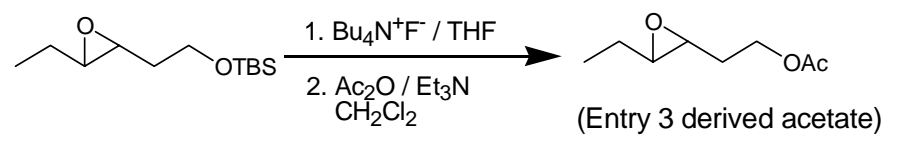

${ }^{1}$ H NMR Conditions: (5 mg) was dissolved in $\mathrm{C}_{6} \mathrm{D}_{6}(0.6 \mathrm{~mL})$, ratio of epoxide $\left.: \mathrm{Eu}(\mathrm{hfc})_{3} \approx 10: 3.3(\mathrm{w} / \mathrm{w})\right\}$

ee measured

Racemic Epoxide<smiles>N=CNCN</smiles>

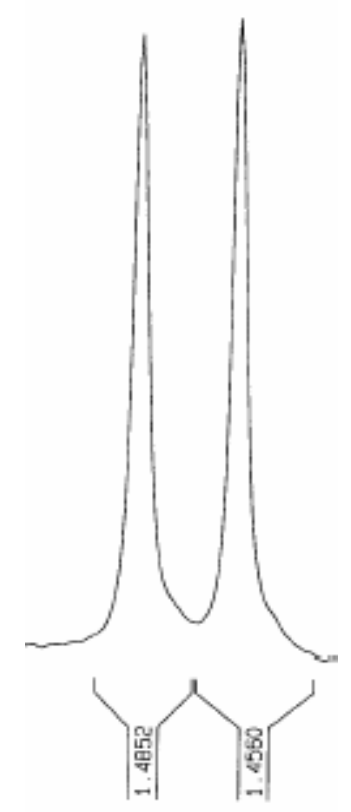

2.3

\section{Chiral Epoxide}
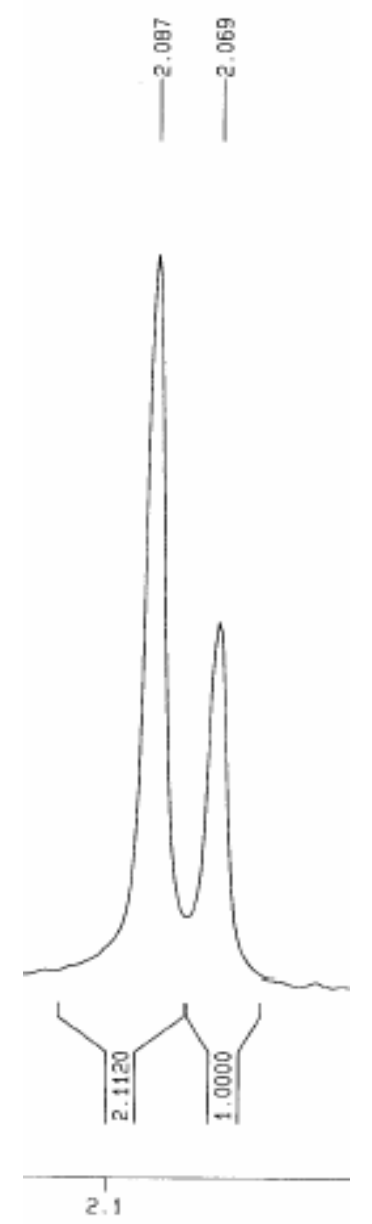


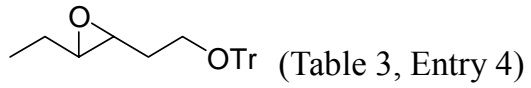

Enantioselectivity was determined after detritylation with Hydrogen and measured its acetate

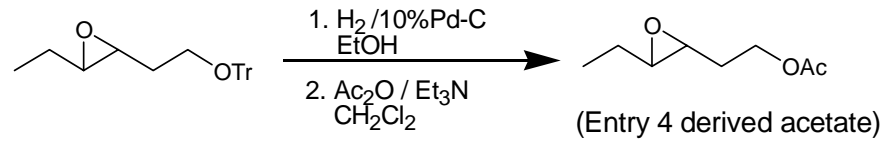

${ }^{1}$ H NMR Conditions: $\quad(5 \mathrm{mg})$ was dissolved in $\mathrm{C}_{6} \mathrm{D}_{6}(0.6 \mathrm{~mL})$, ratio of epoxide $\left.: \mathrm{Eu}(\mathrm{hfc})_{3} \approx 10: 3.3(\mathrm{w} / \mathrm{w})\right\}$

ee measured

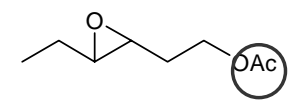

Racemic Epoxide<smiles>N#CNCN</smiles>

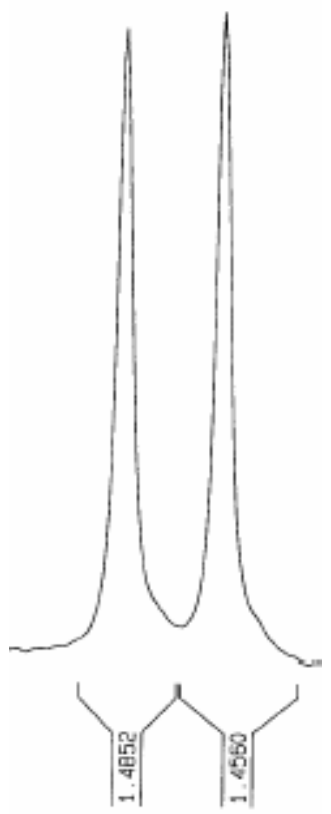

2.3
Chiral Epoxide

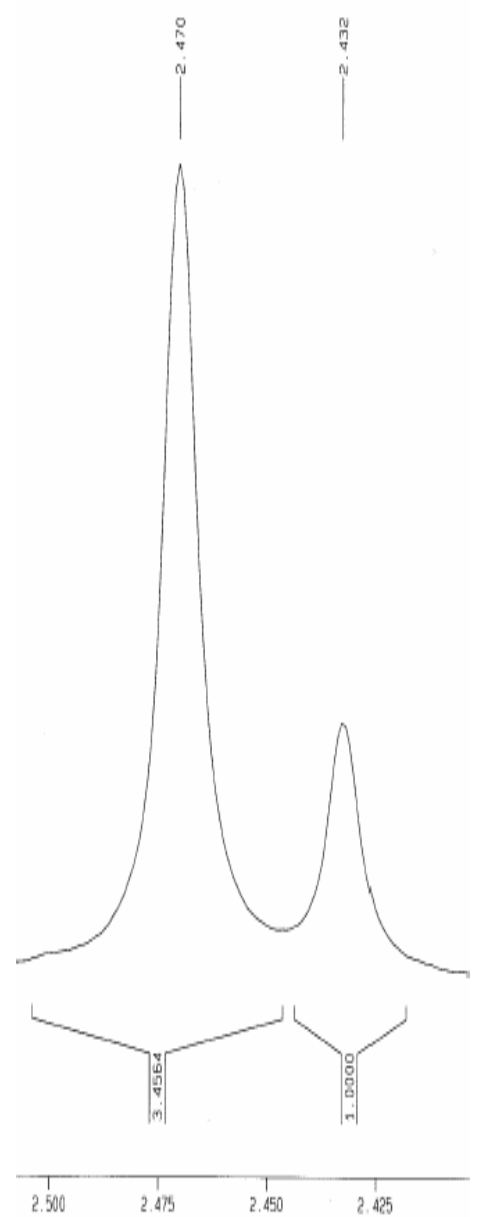




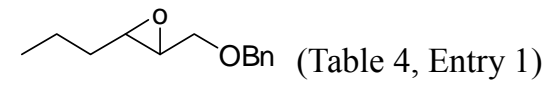

HPLC Conditions: Column: Chiralcel OD-H.

Eluent: Hexane/2-propanol (90/10); Flow rate: $0.5 \mathrm{ml} / \mathrm{min}$;

Detection:UV 254nm

\section{Racemic Epoxide}

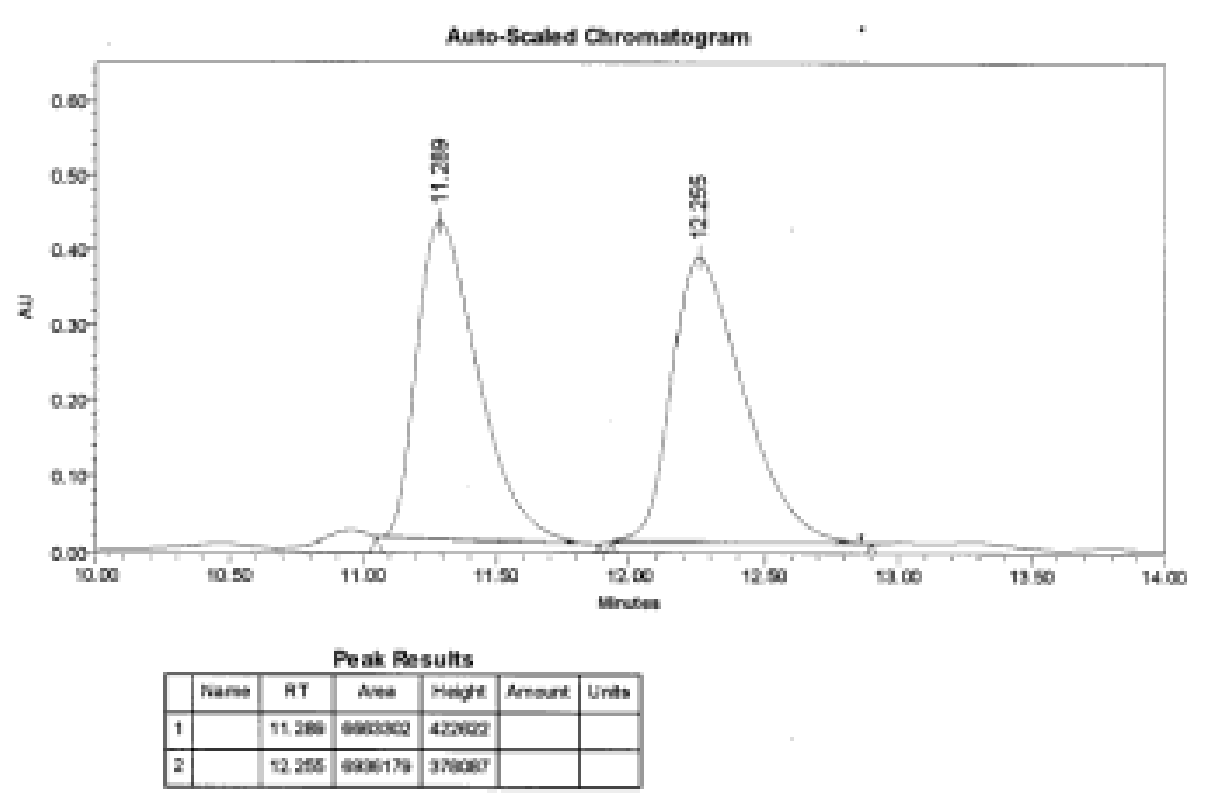

\section{Chiral Epoxide}

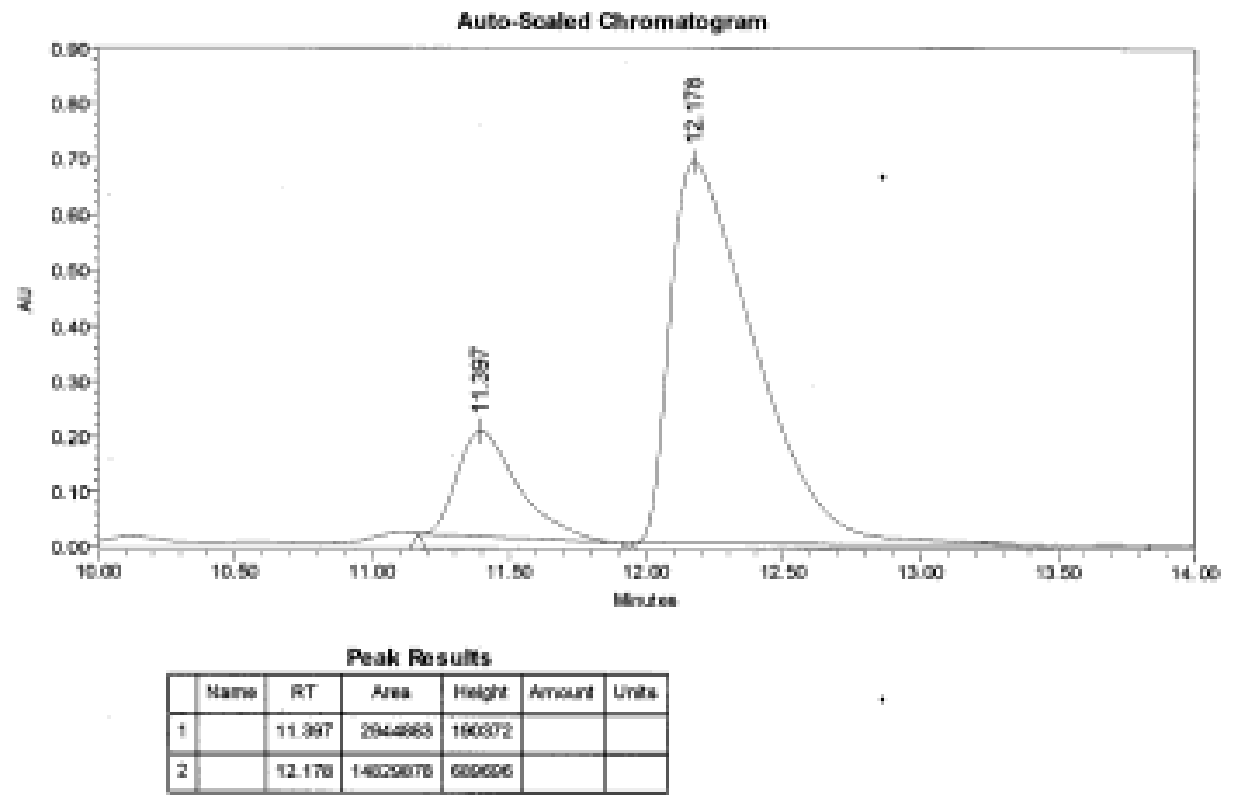




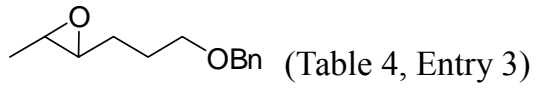

HPLC Conditions: Column: Chiralcel OD-H.

Eluent: Hexane/2-propanol (99/1); Flow rate: $0.5 \mathrm{ml} / \mathrm{min}$;

Detection:UV 254nm

\section{Racemic Epoxide}

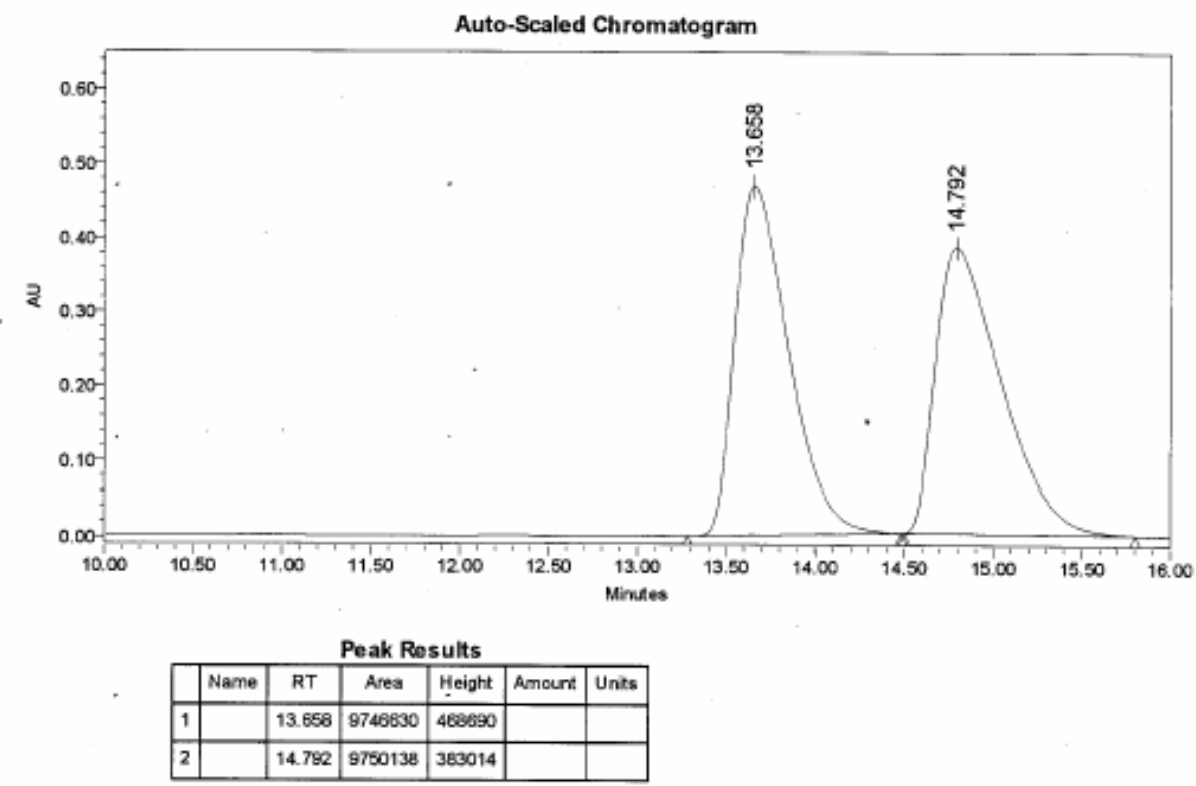

\section{Chiral Epoxide}

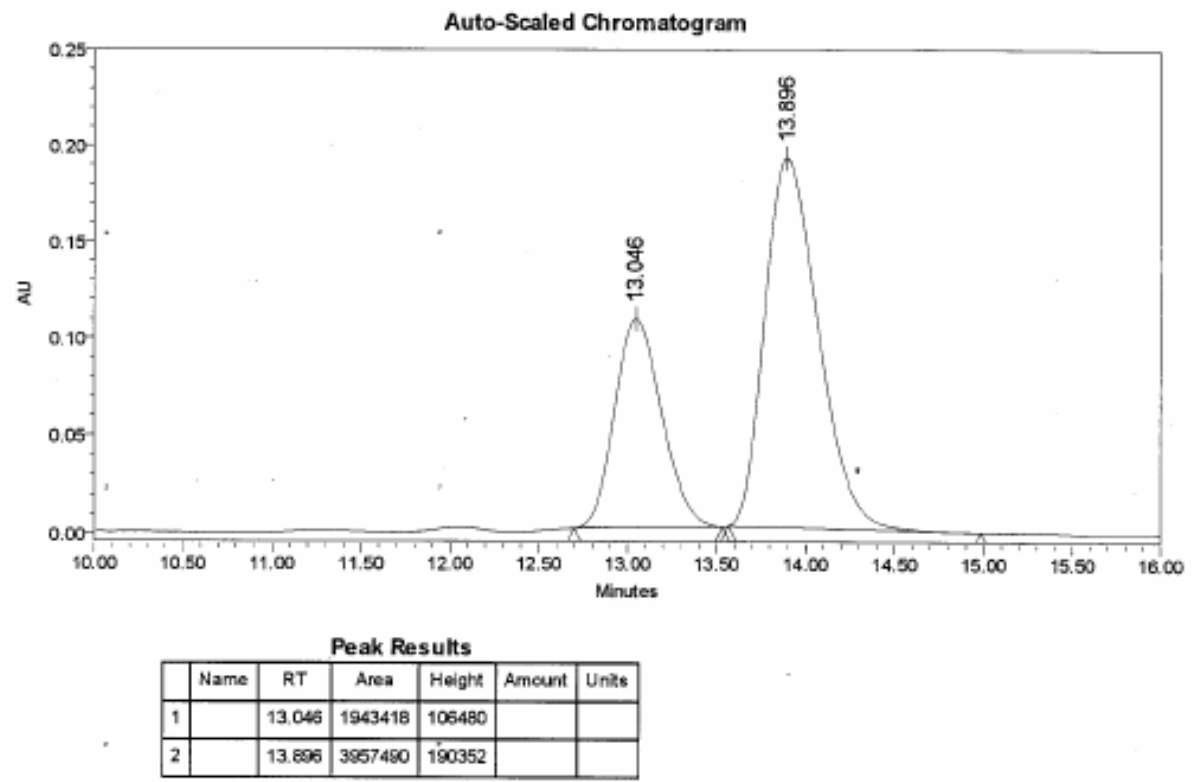



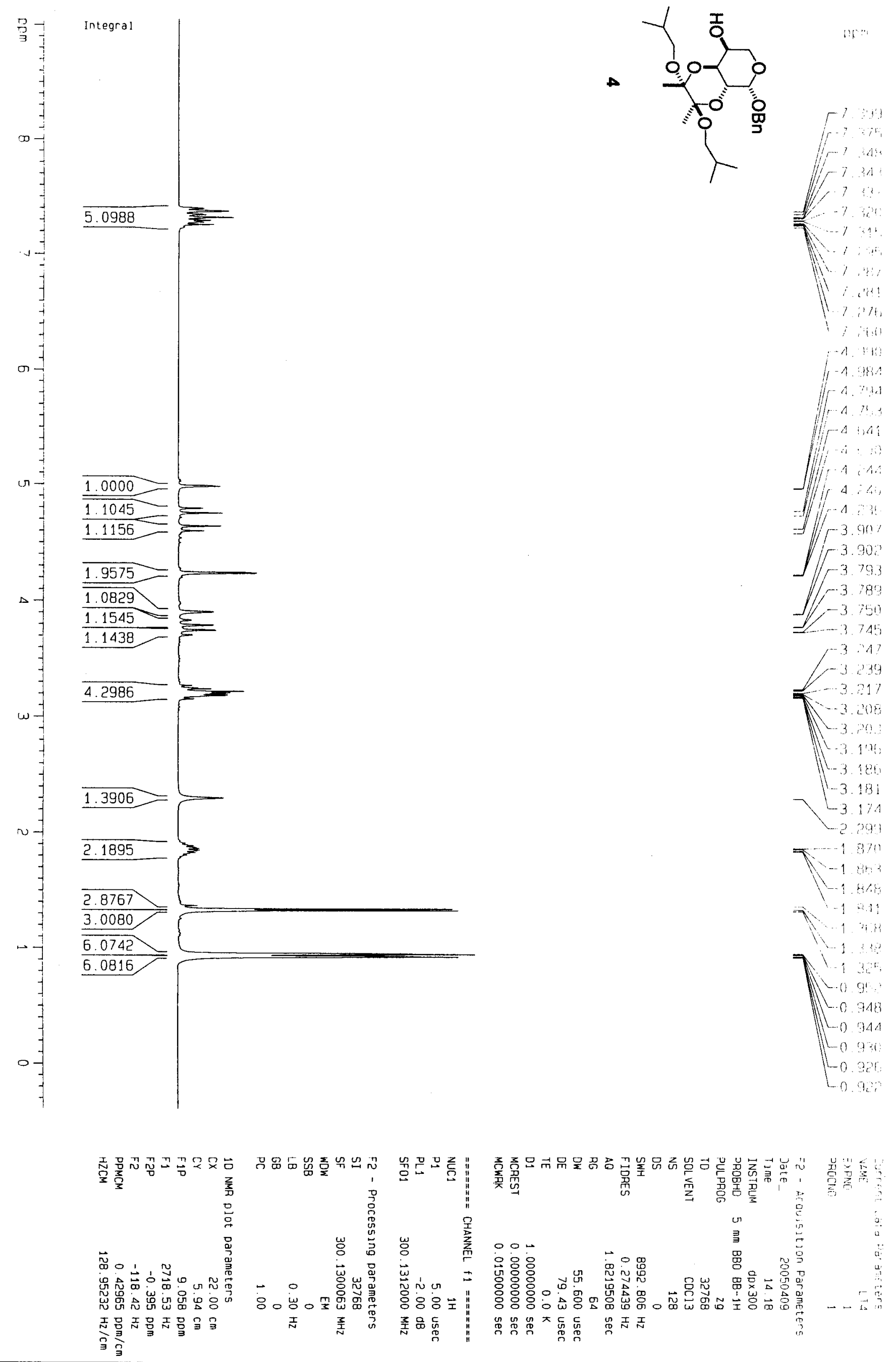

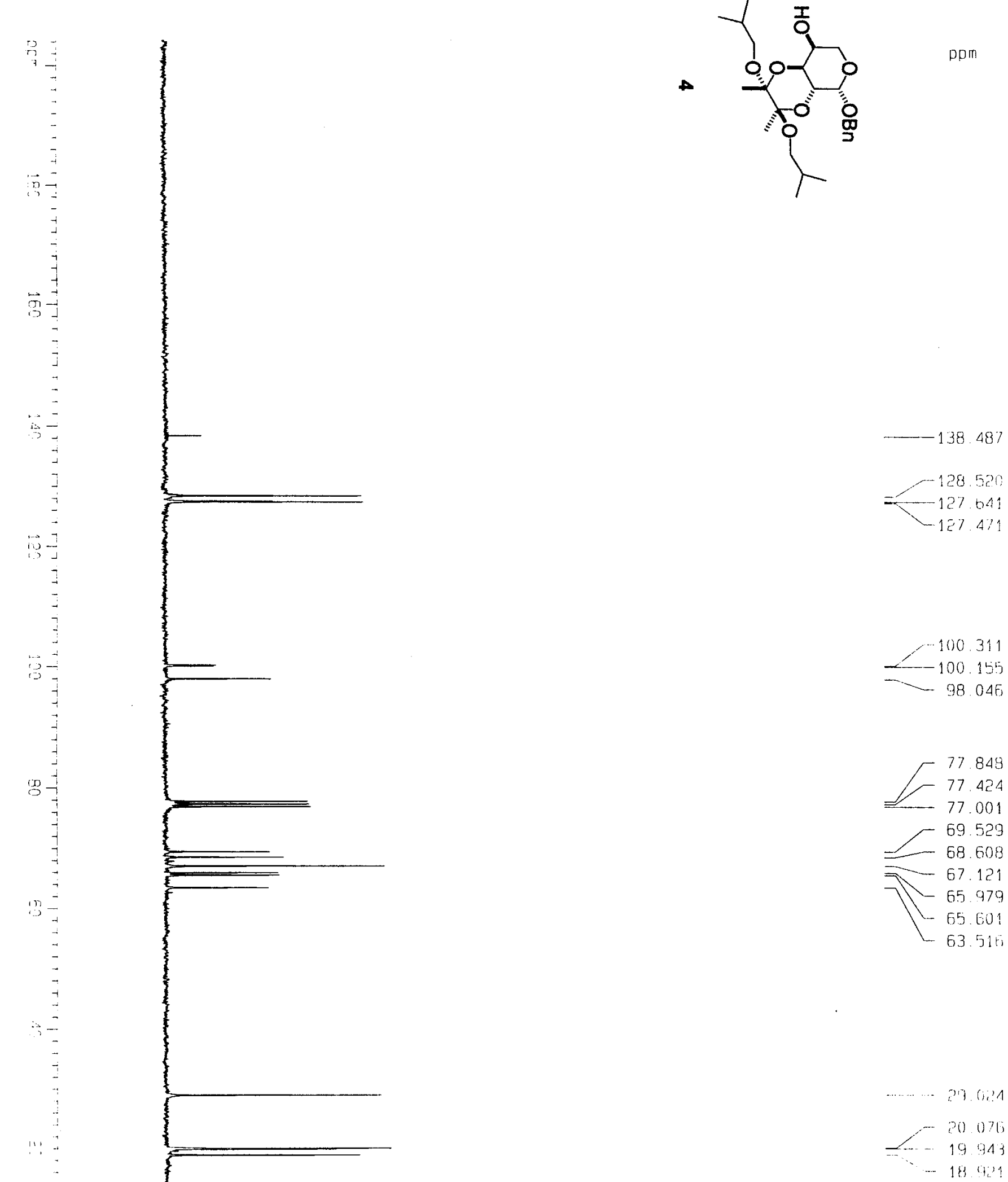

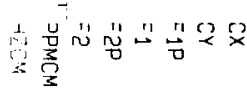

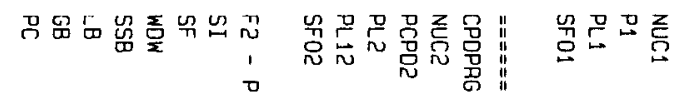

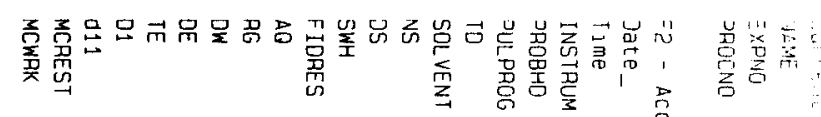

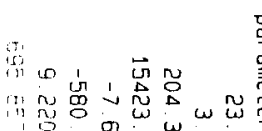

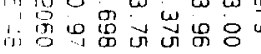

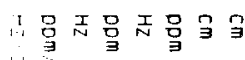

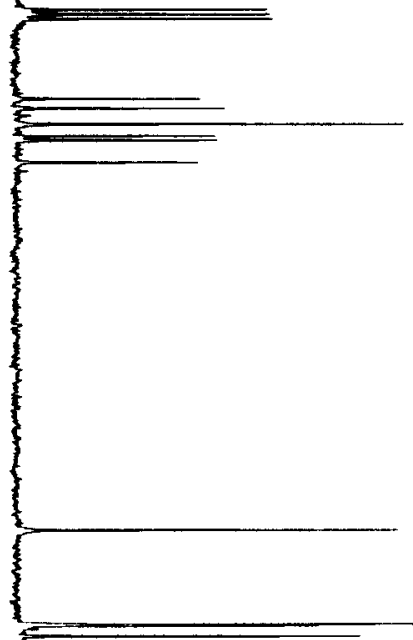

1843 

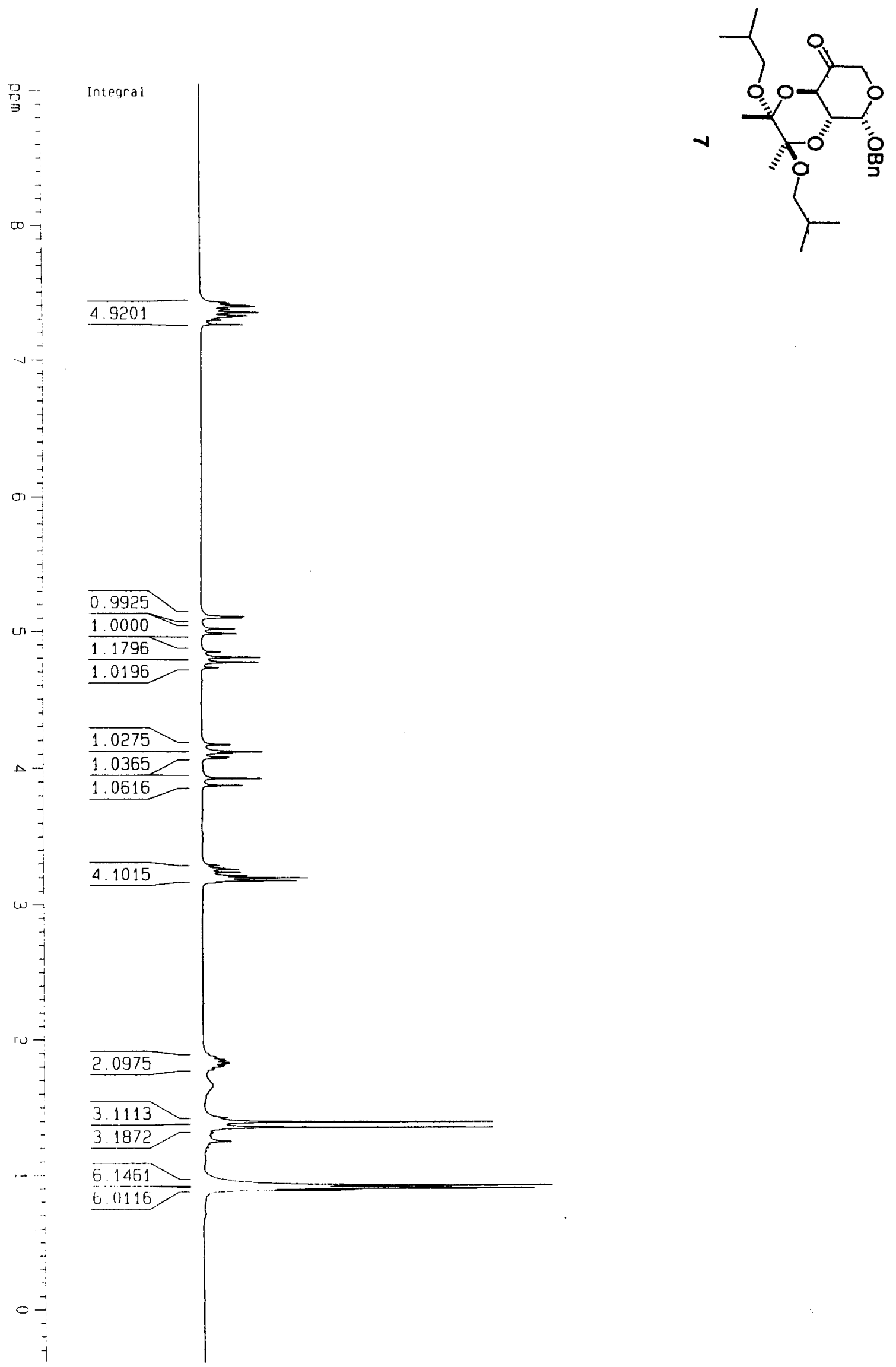

ppm

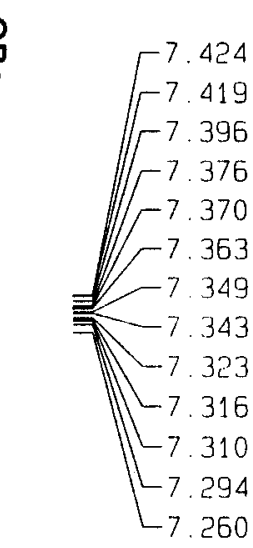

5.112

$-5.102$

$-5.019$

$-4.983$

$-4.847$

$-4.806$

$\Gamma^{-4.771}$

I.170

$=\Gamma^{4.118}$

$\Gamma^{4.106}$

$-4.080$

$-4.070$

$-3.925$

$-3.875$

$\Gamma^{3.268}$

3.259

$-3.238$

$-3.213$

$-3.197$

$-3.191$

$-3.183$

$L_{3.175}$

$-3.161$

$\digamma^{1.856}$

$-1.834$

$-1.825$

$-1.812$

₹ -1.803

$9-1.429$

$\begin{array}{ll}-1 & 394\end{array}$

$7-1.379$

- 1.1 .35

$L_{1} .339$

$L_{1} .252$

$L_{0.925}$

$-0.909$

$\left[\begin{array}{r}0.903 \\ 0.887\end{array}\right.$

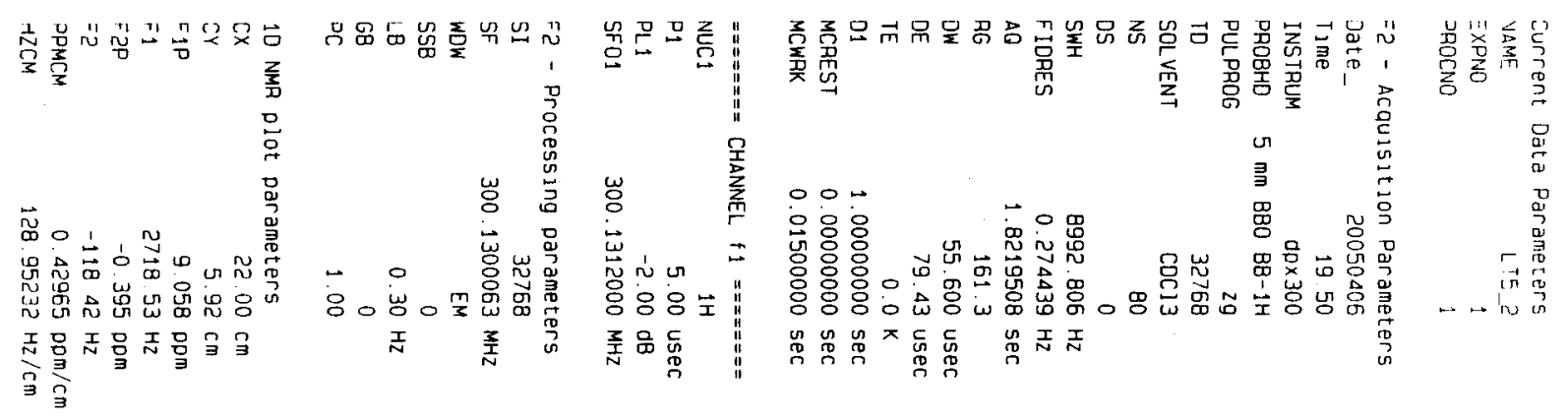




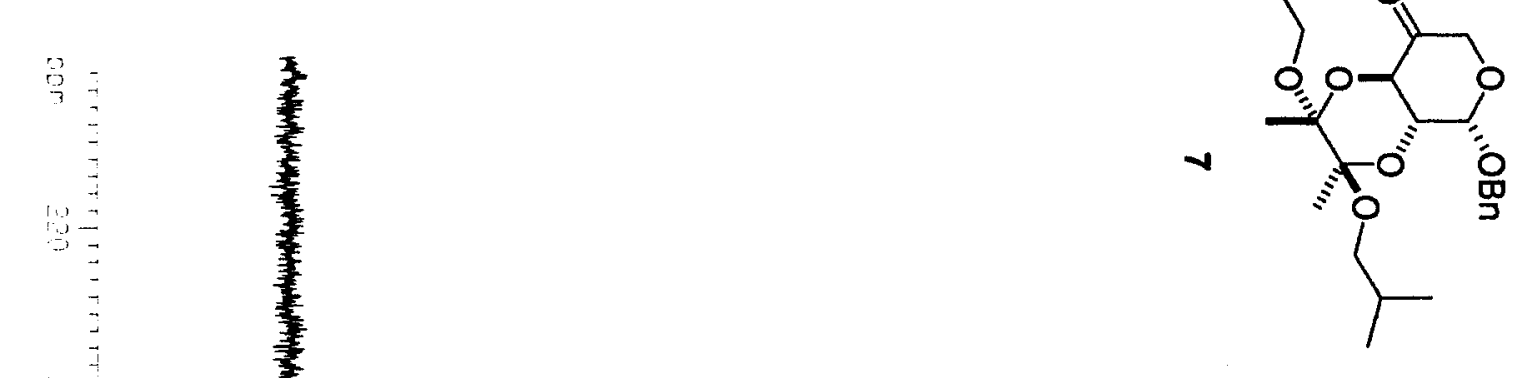

$-200.91$
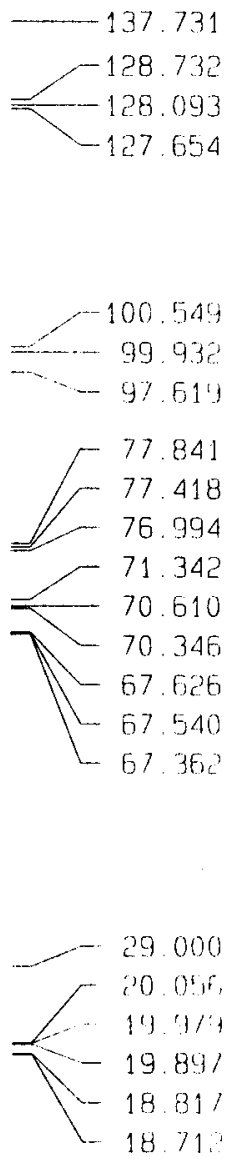

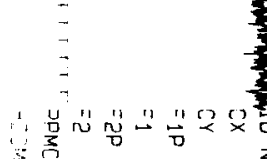

要 0

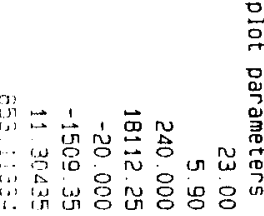

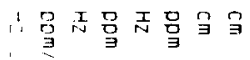

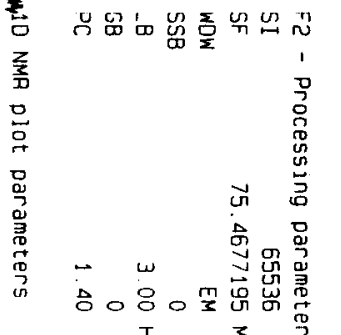

工 蛋 总

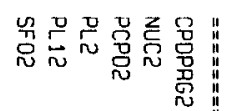

岩

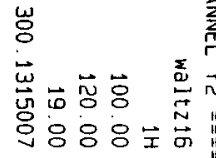

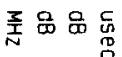

苞믐몽

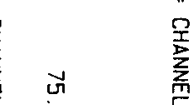

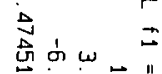

58 욤

존 吕点

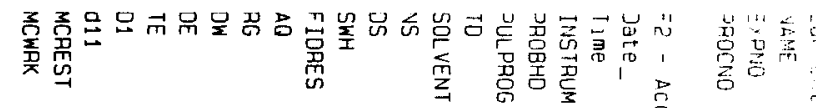

$\begin{array}{lll}0 & 0 \\ 0 & 0 & 0 \\ 0 & 0 \\ 0 & 0\end{array}$

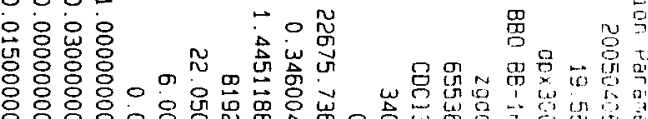

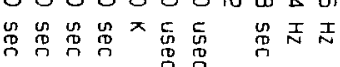




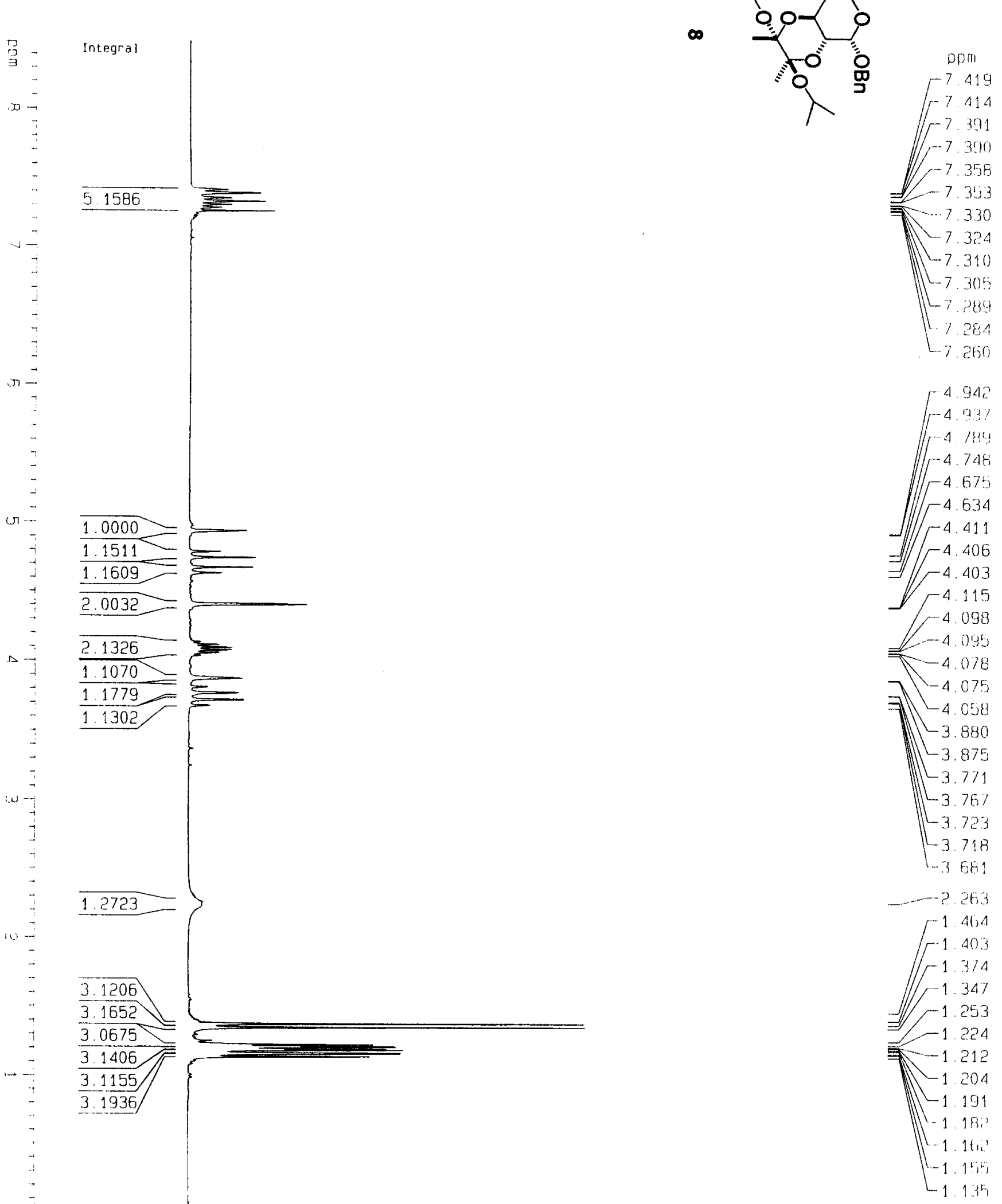

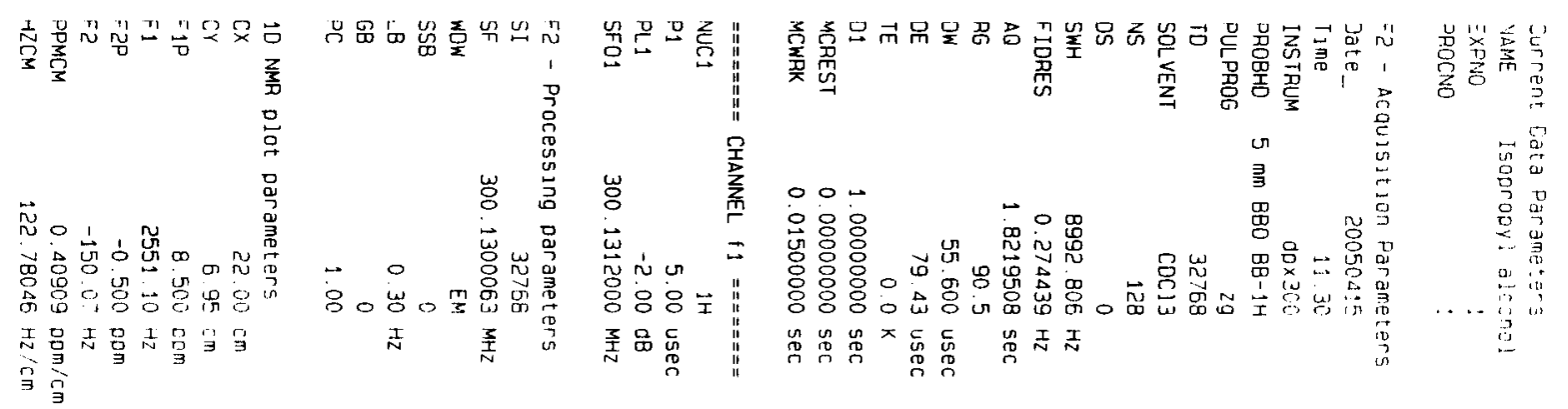




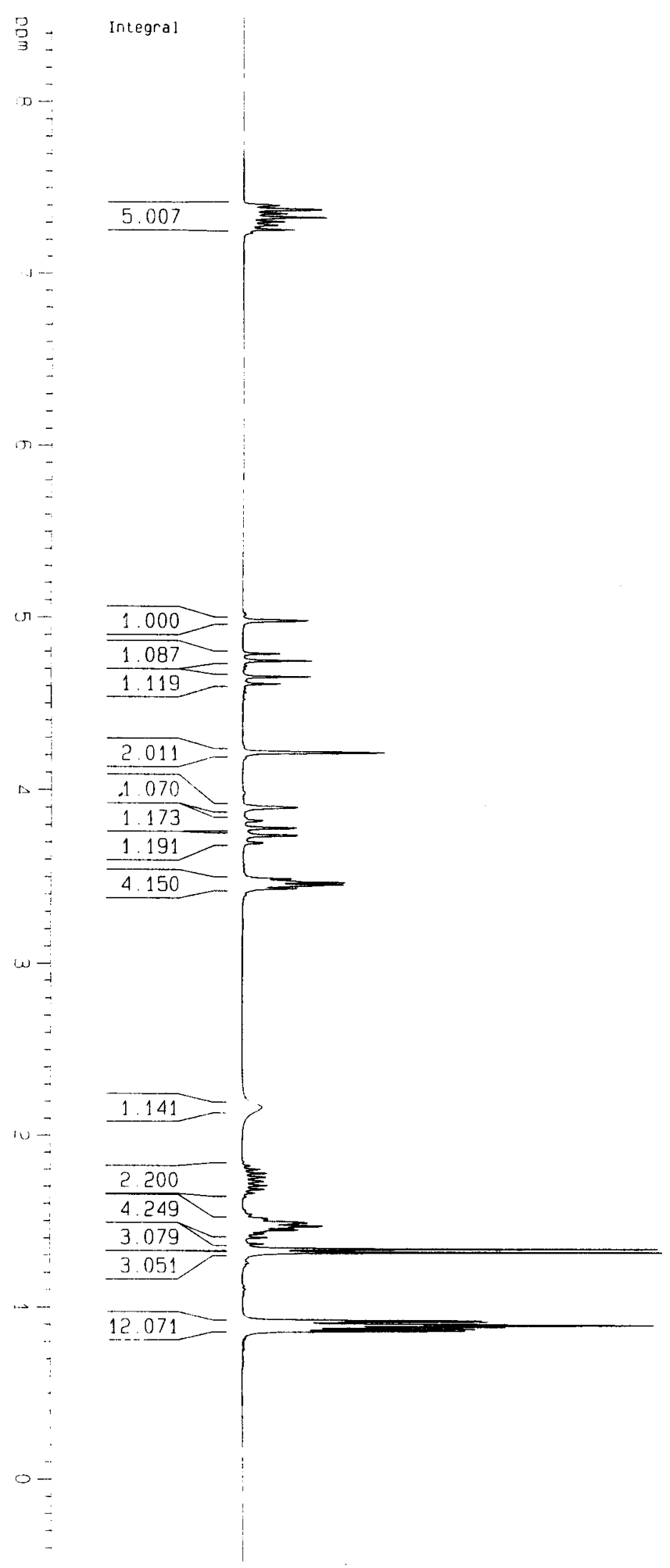

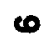
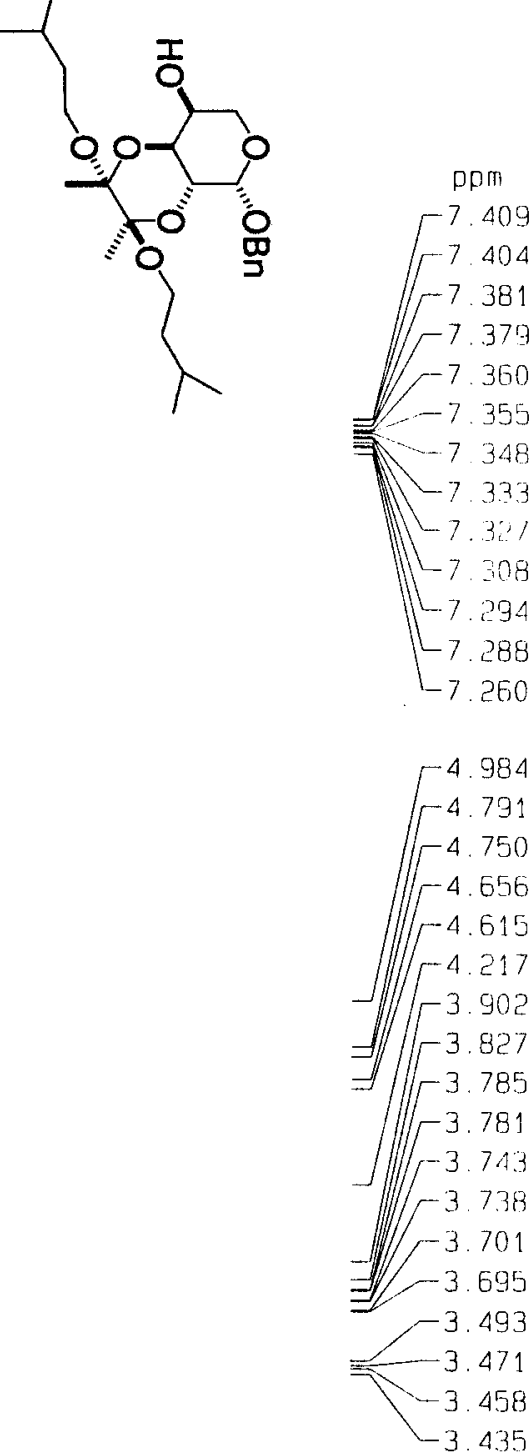

$-2.171$

$\Gamma^{1} .778$

$-1.706$

-1.510

$-1.504$

] $[-1.494$

$-1.488$

$-1.482$

$-1.471$

$-1.468$

$-1.459$

$-1.447$

$-1.337$

$-1.317$

M-0 0

$-0.915$

$(0.901$

(-0.834

$L_{0.884}$

$-0.873$

$L_{0.862}$

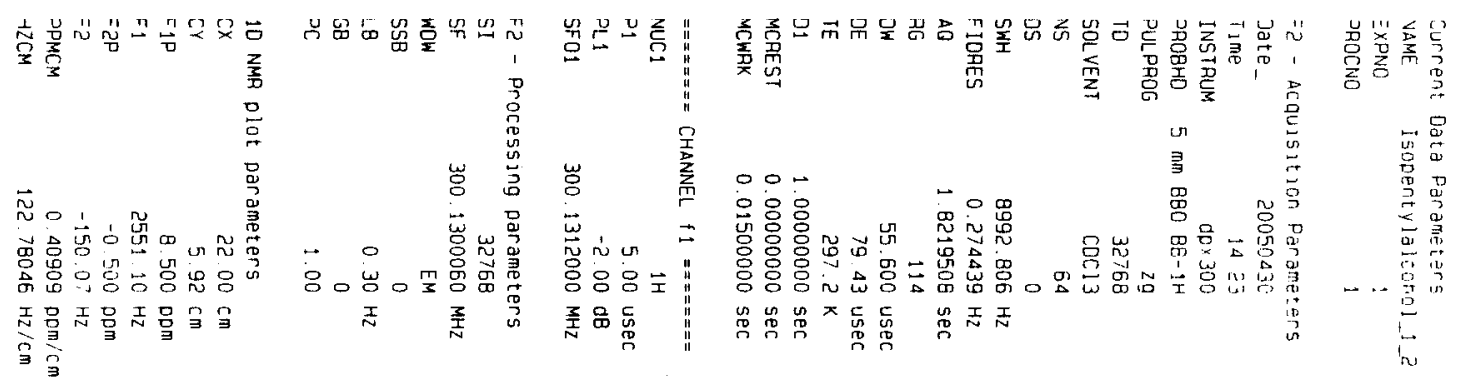




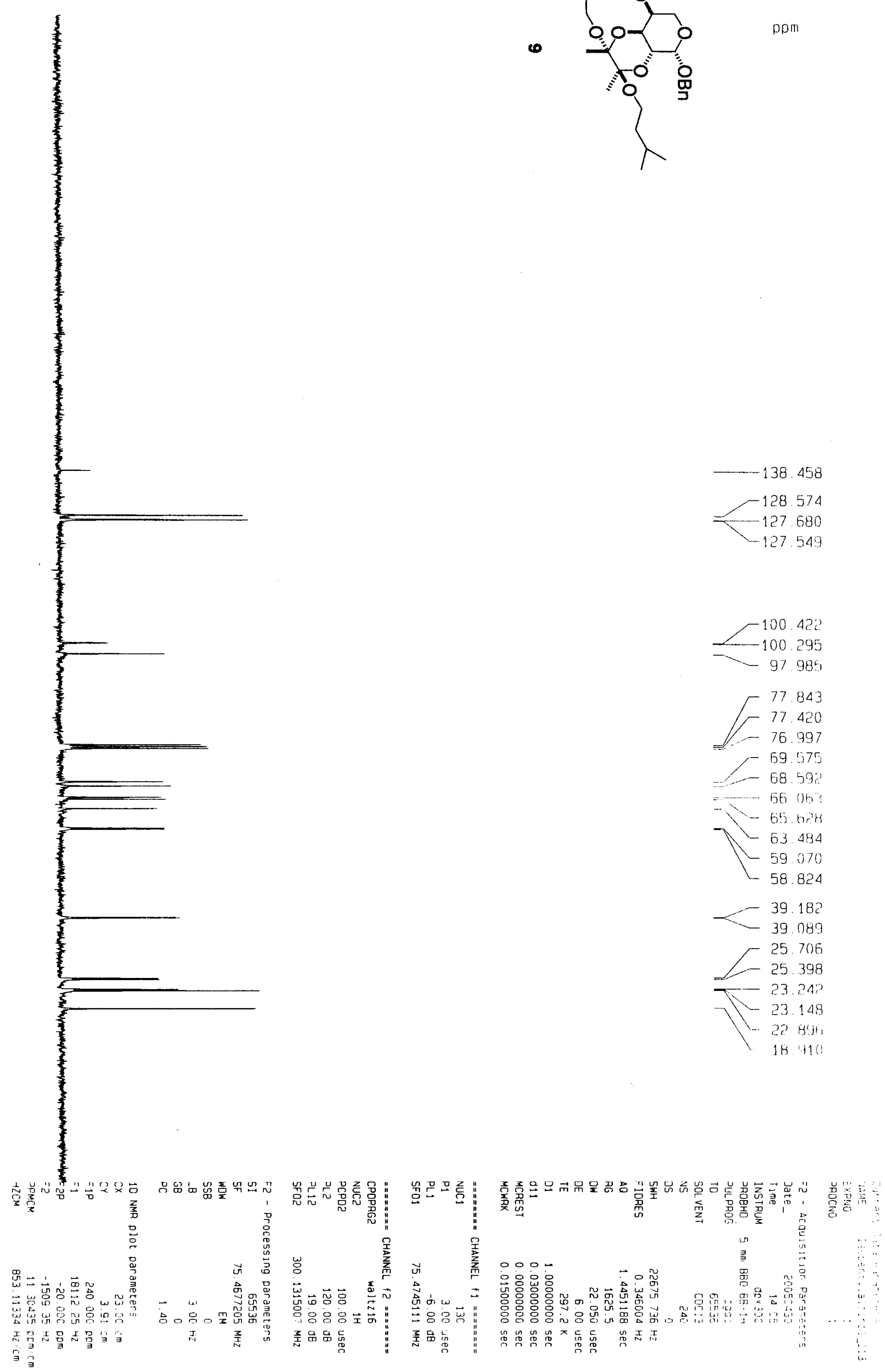



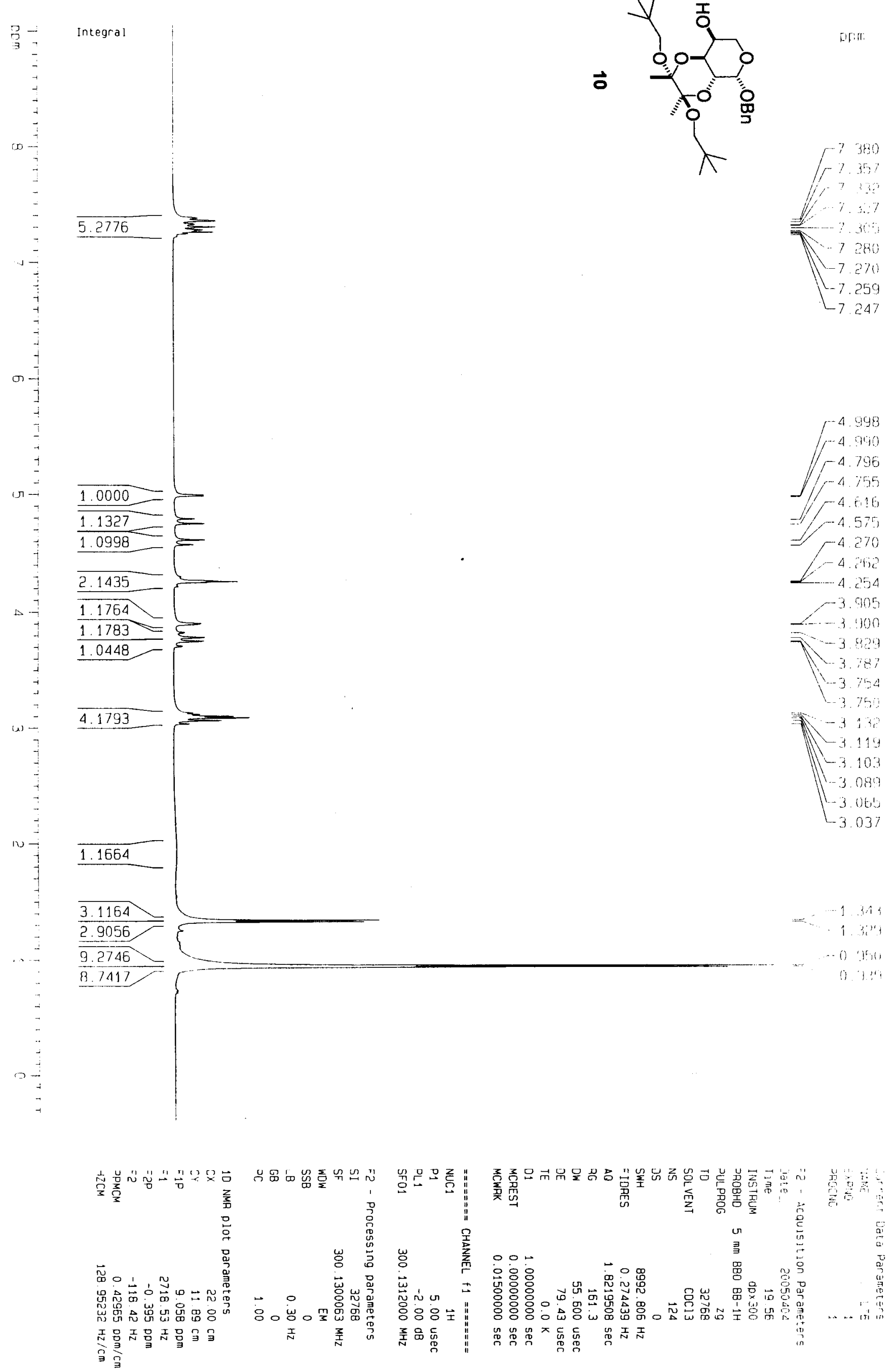


$$
1
$$



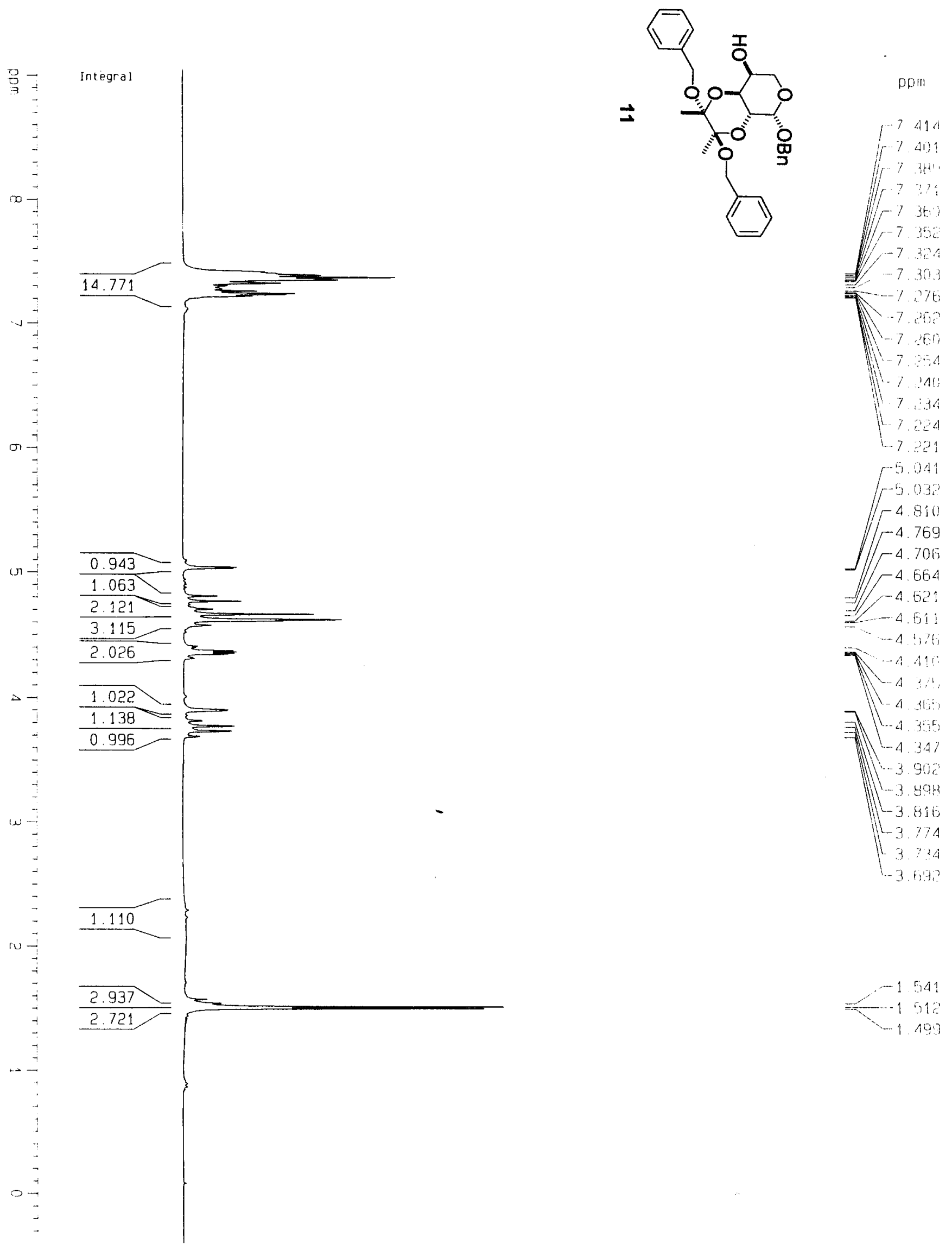

$-154$

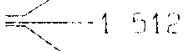

$-1 .-190$

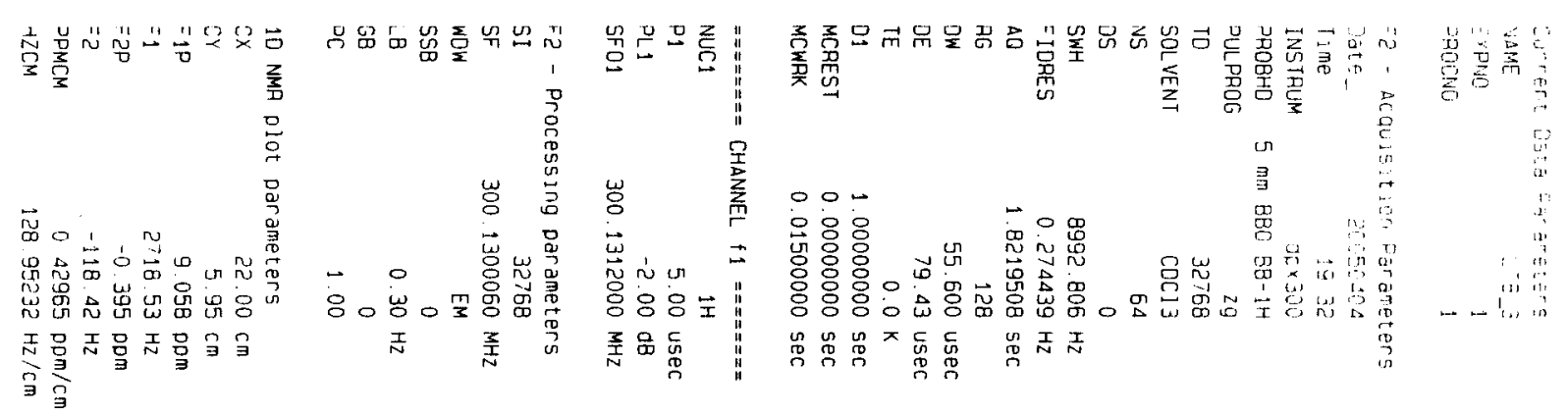



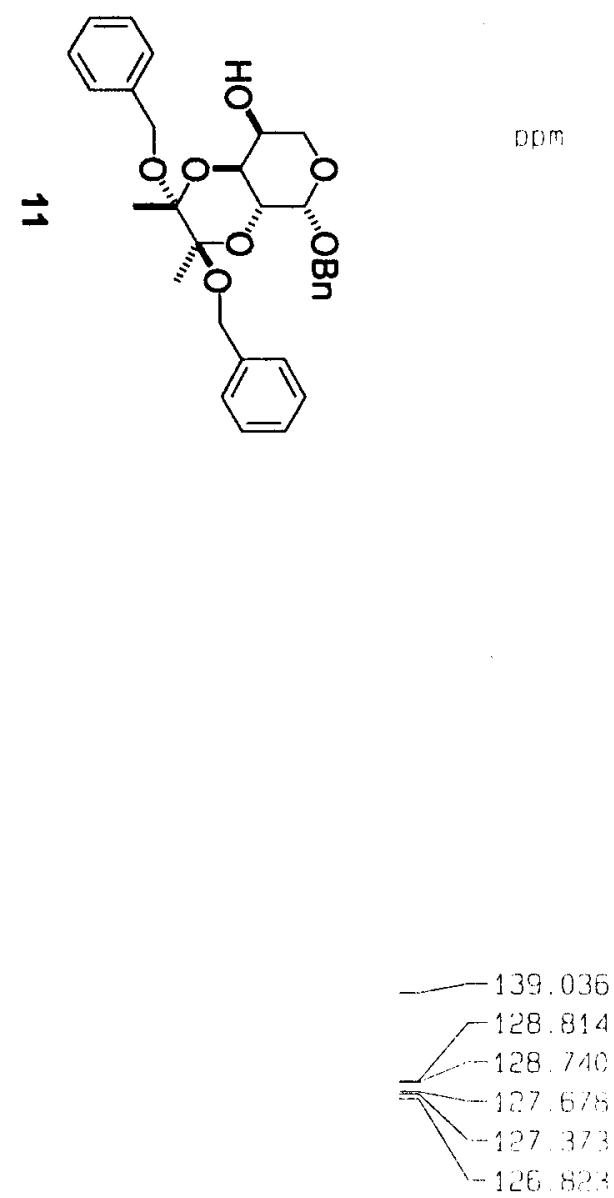

$-100.812$

$-\quad 97.939$

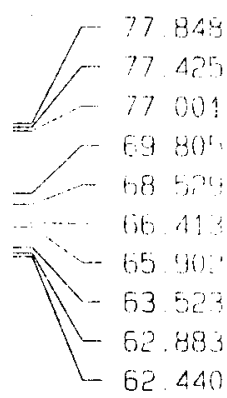

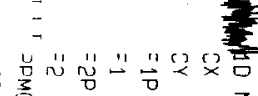

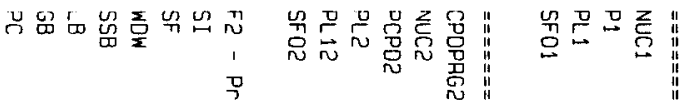

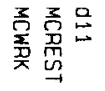



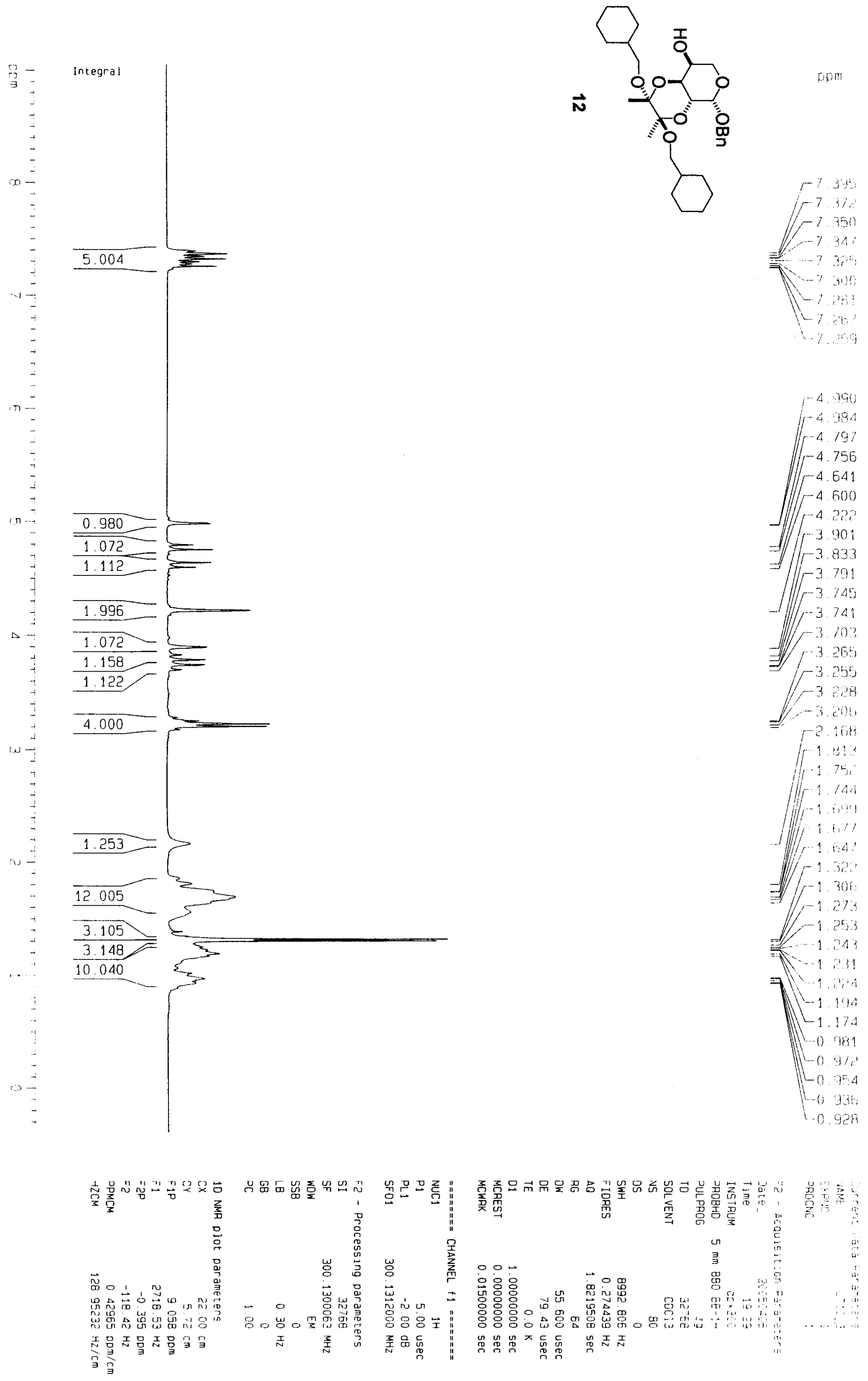


$$
=
$$



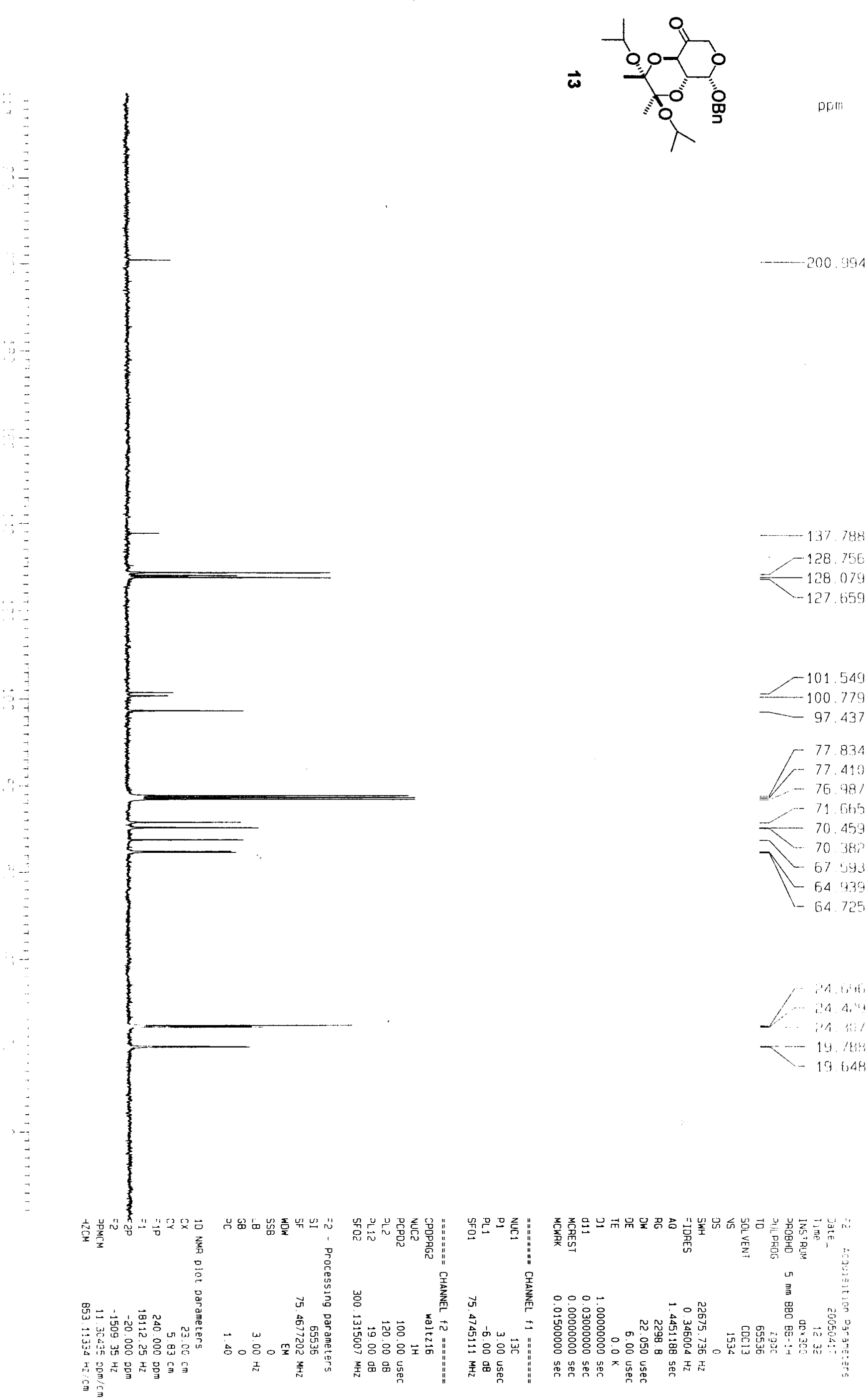

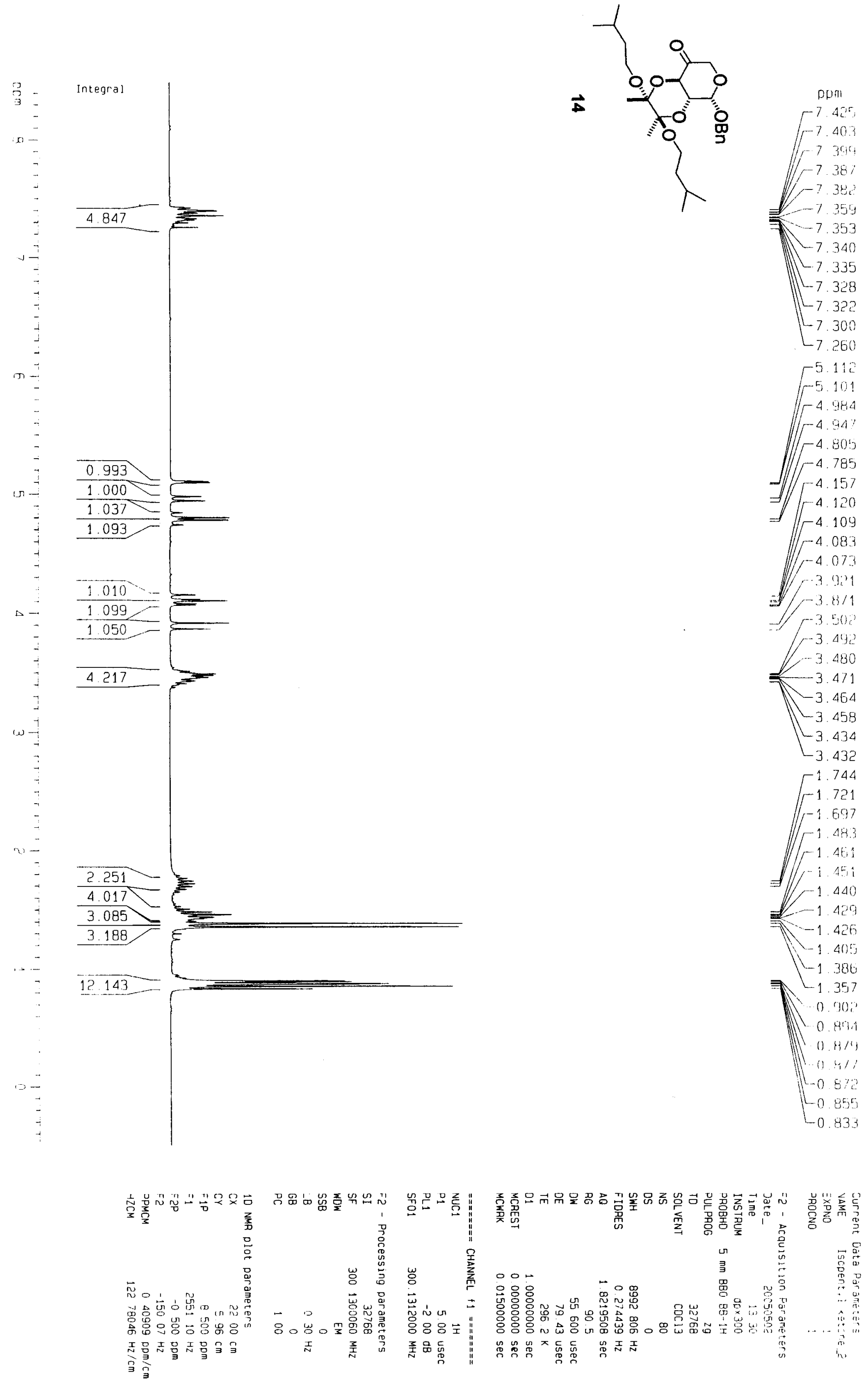


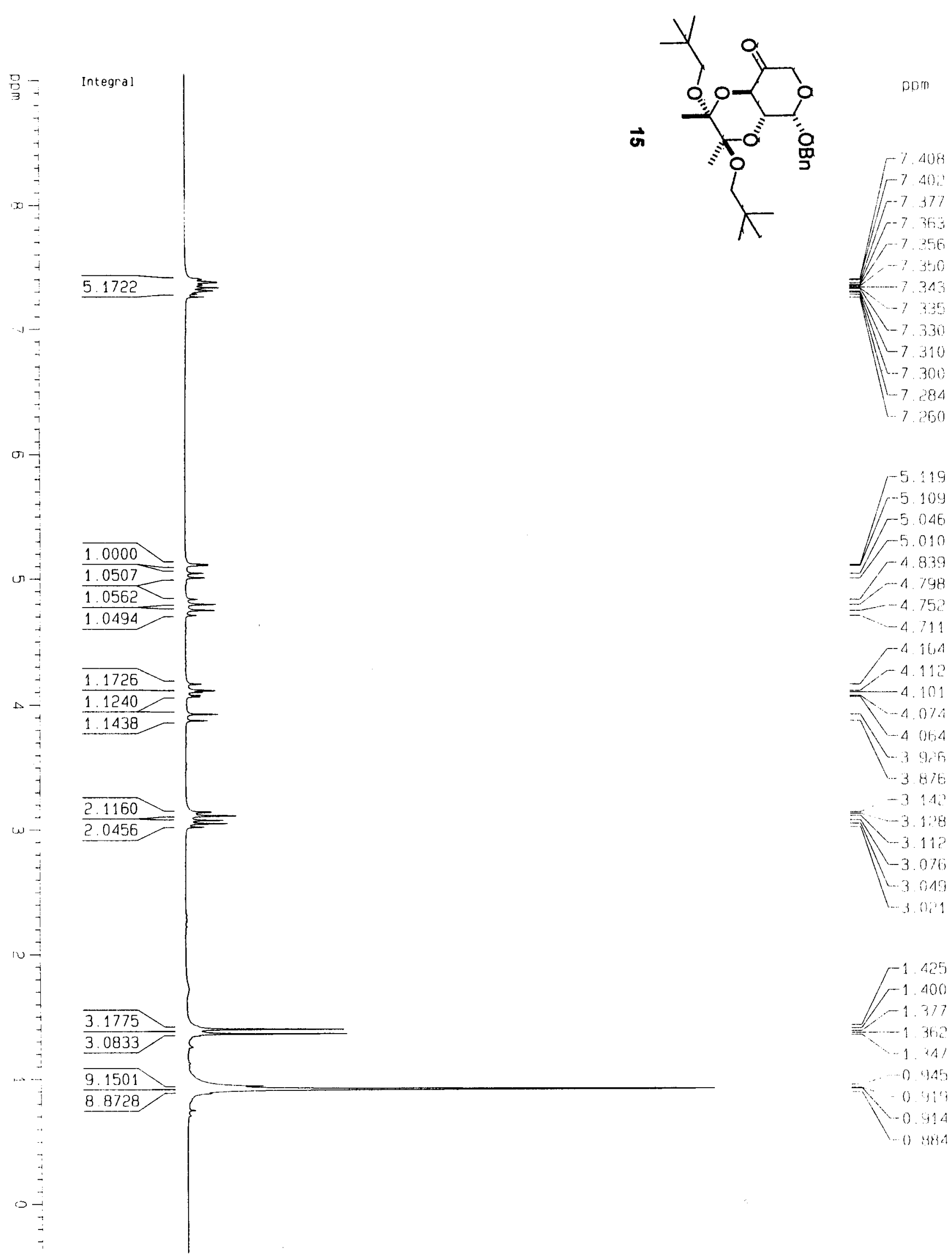

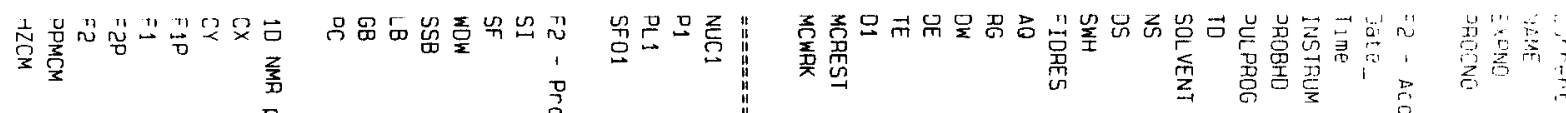

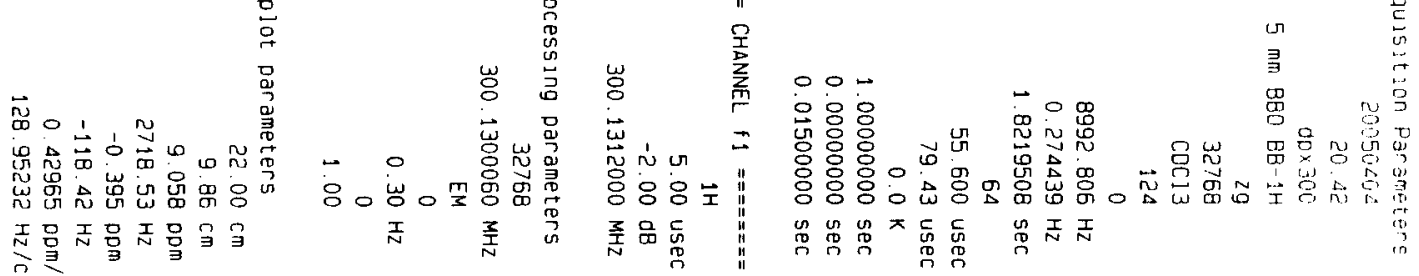




$$
=
$$



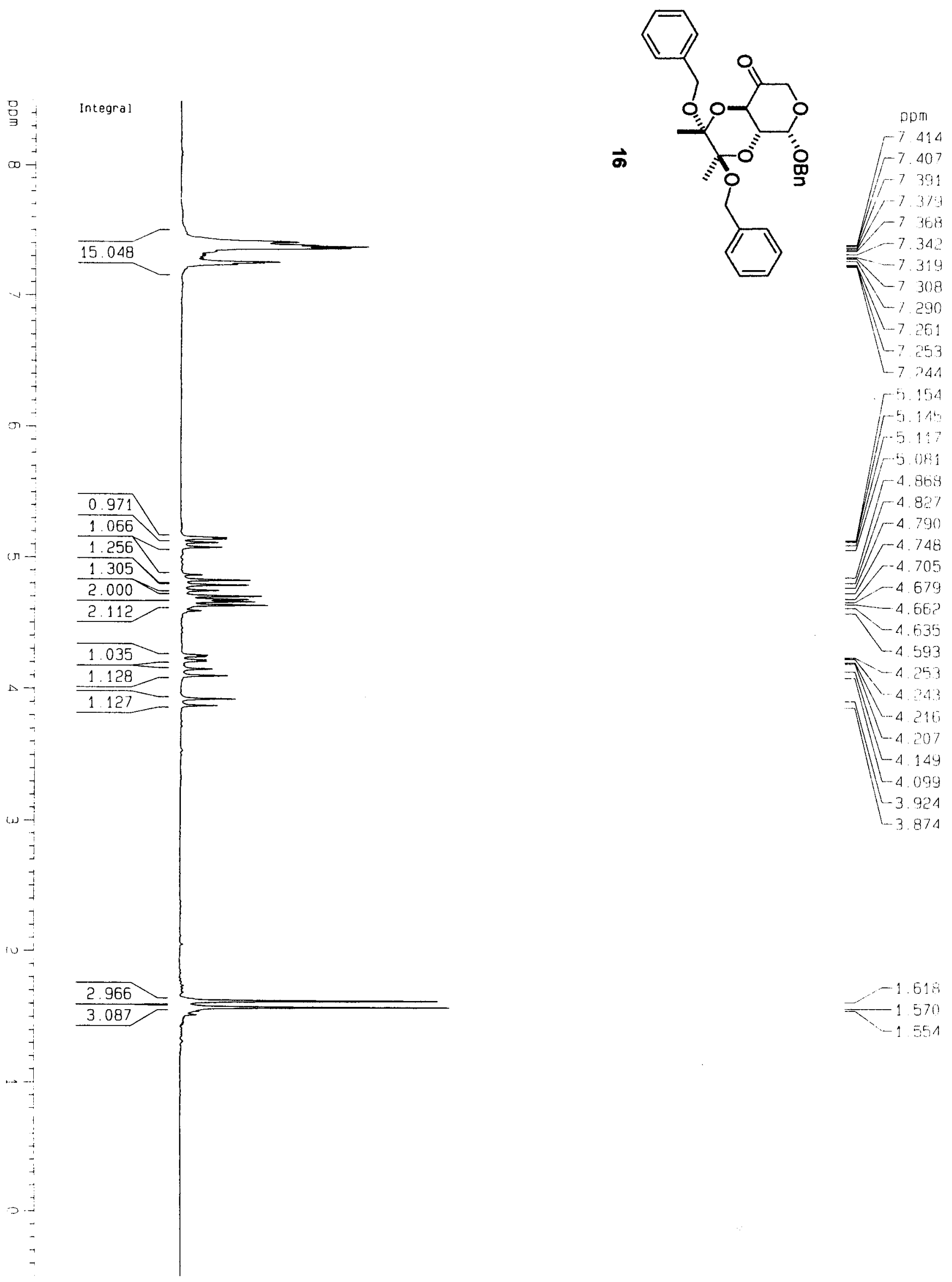

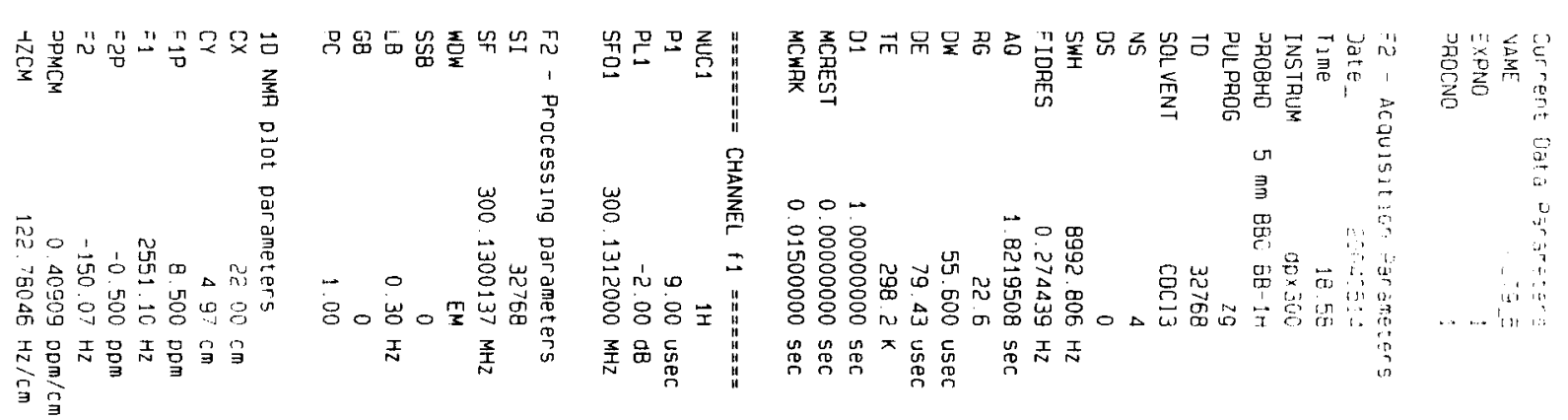




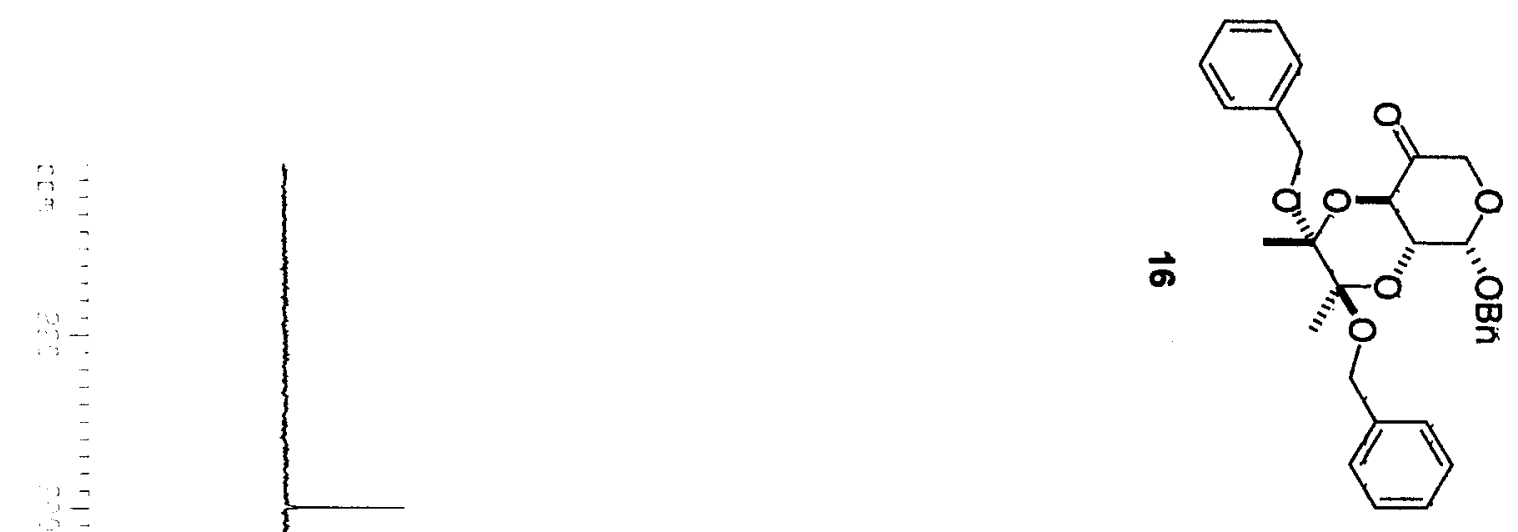

$-200 \cdot 120$
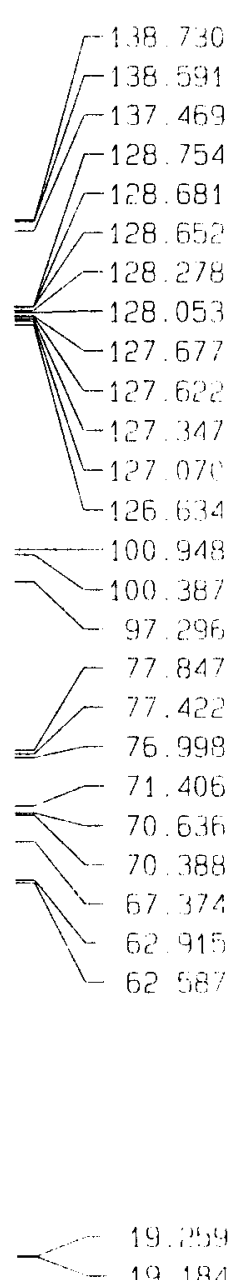

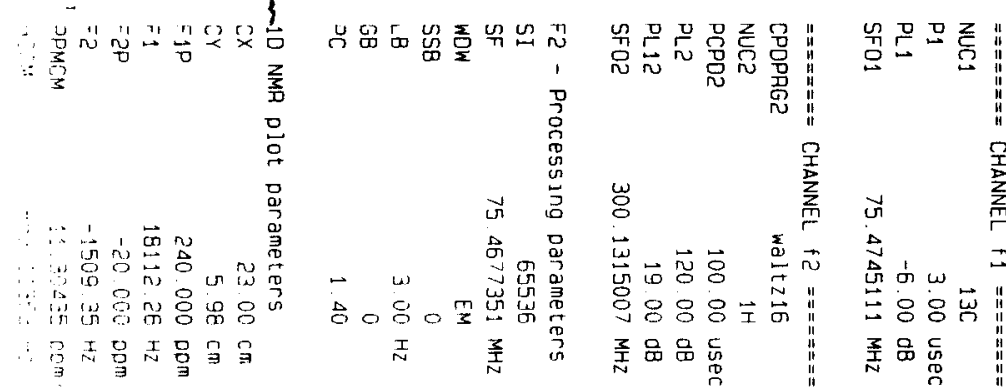

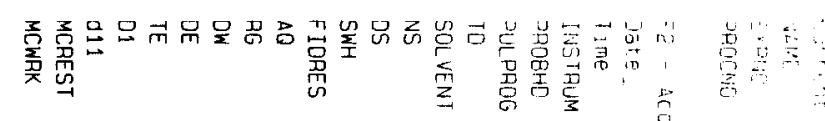

$000-$

$\begin{array}{lll} & \\ 0 & 0 \\ 0 & 0 & 0 \\ 0\end{array}$ 

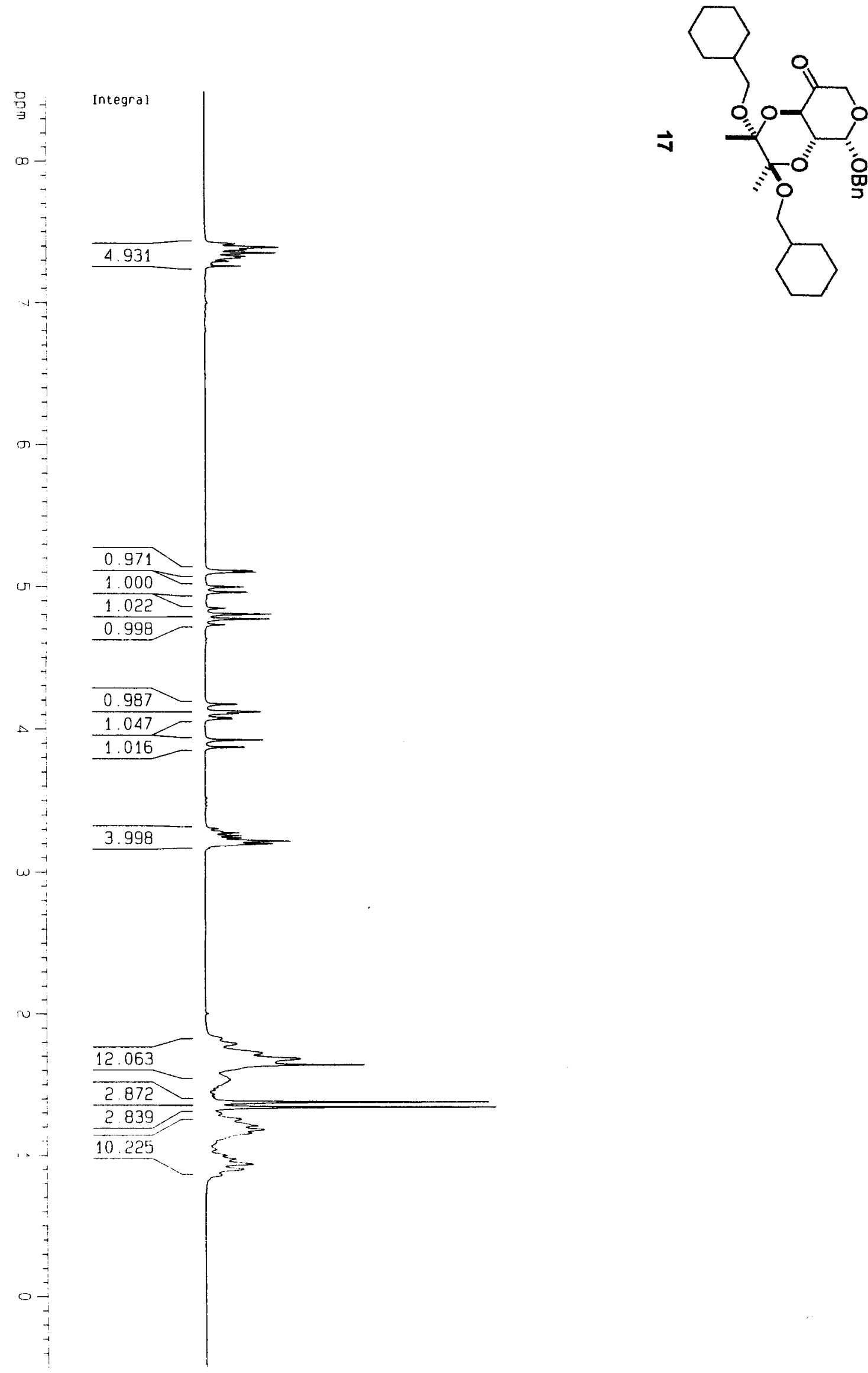

ppm
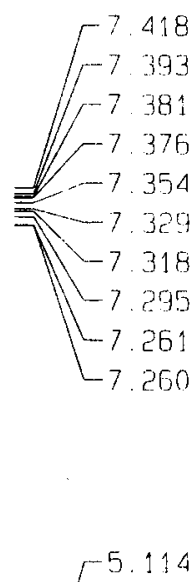

$-5.104$

$-4.959$

$\sqrt{-4 \cdot 962}$

$-4.848$

$1 / f^{-4.807}$

-4.733
-4.732

4.172
-4.119
-4.108

$-4.081$

$-4.071$

$-3.924$

$-3874$

$--3.277$

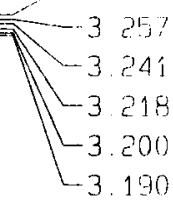

1.787
-1.772

$r^{-1.72 ?}$

$F^{-1.68}$

$-1.639$

$-1.53$

1.377

$=-1341$

- 1.100

$7(-1.180$

$(-1.155$

$-1.000$

$-0.973$

$L_{0}, 96$

$L_{0}-936$

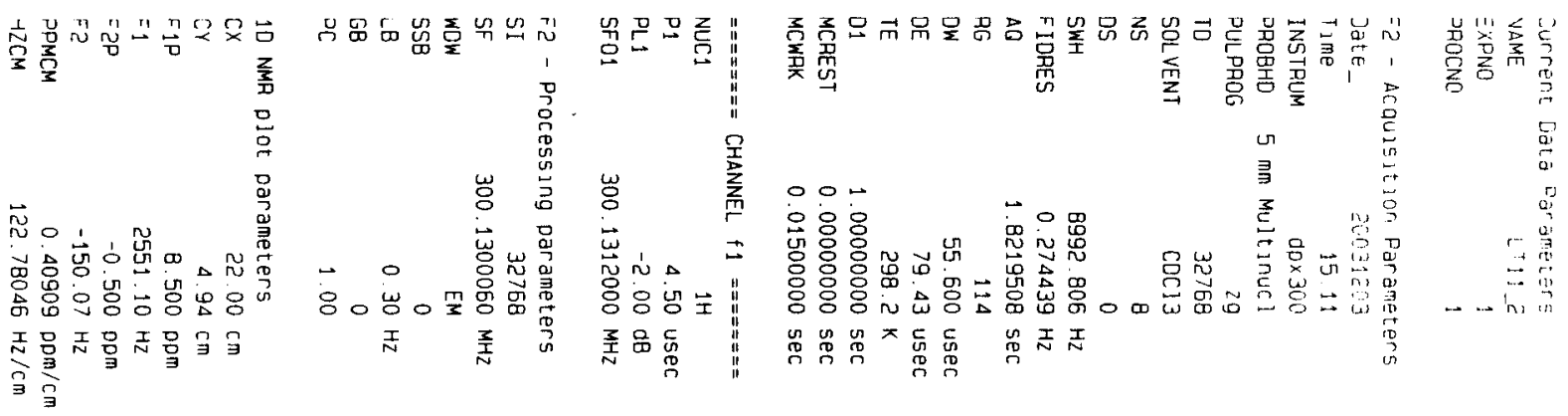




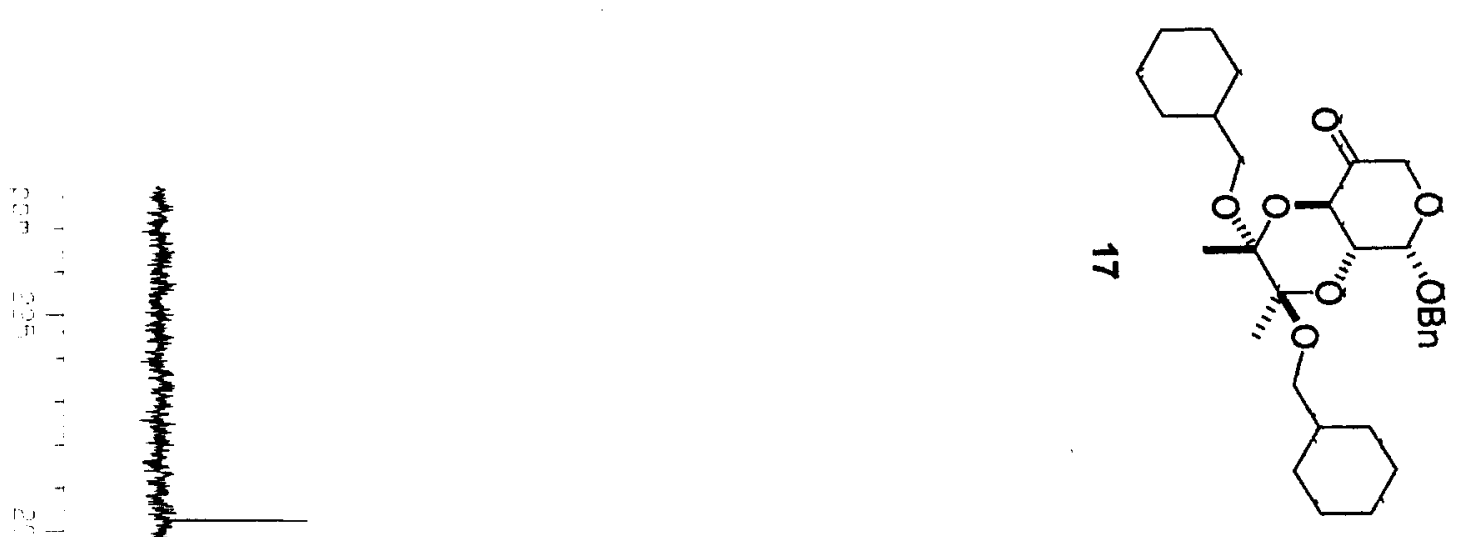

S46

$-20.201 .277$
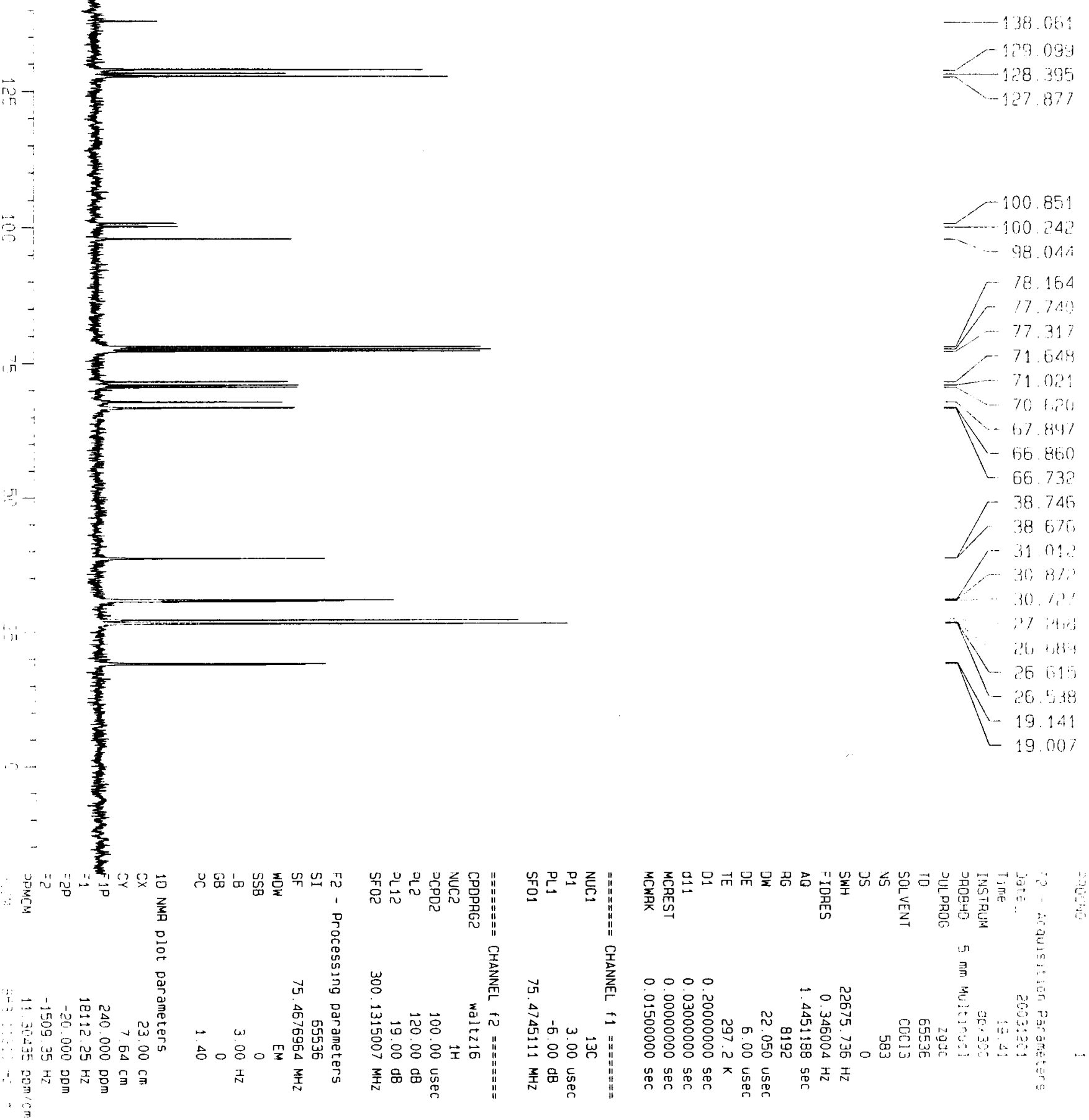

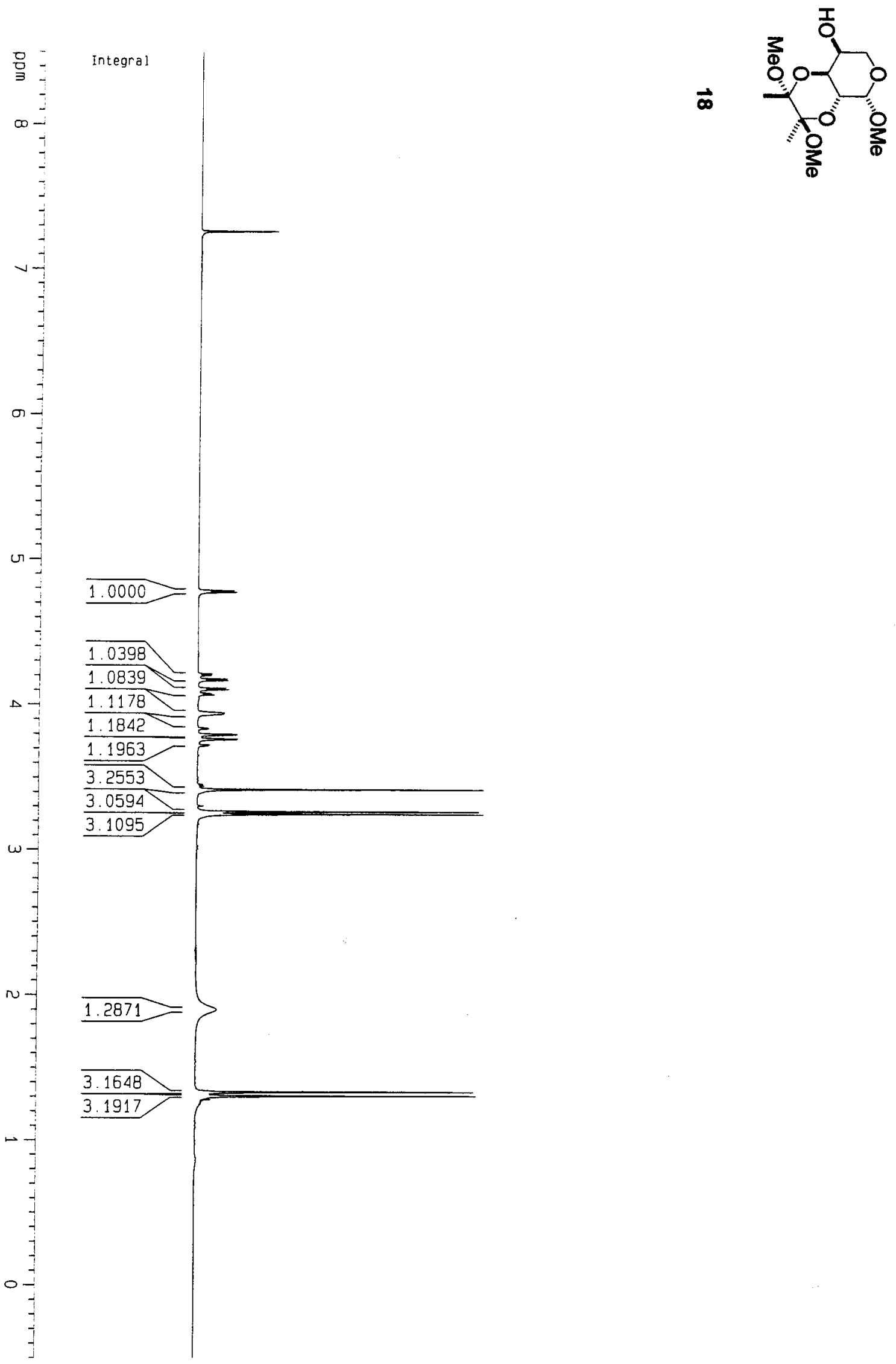

ppm
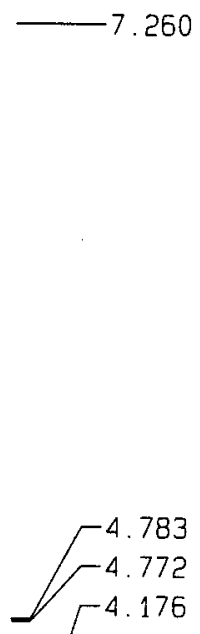

$r^{4.165}$

4.112

$\Gamma^{4.101}$

3.944

$-3.934$

$-3.796$

$-3.792$

$-3.765$

$-3.760$

$-3.417$

$-3.264$

$-3.245$

$-1.900$

-1.332
-1.304

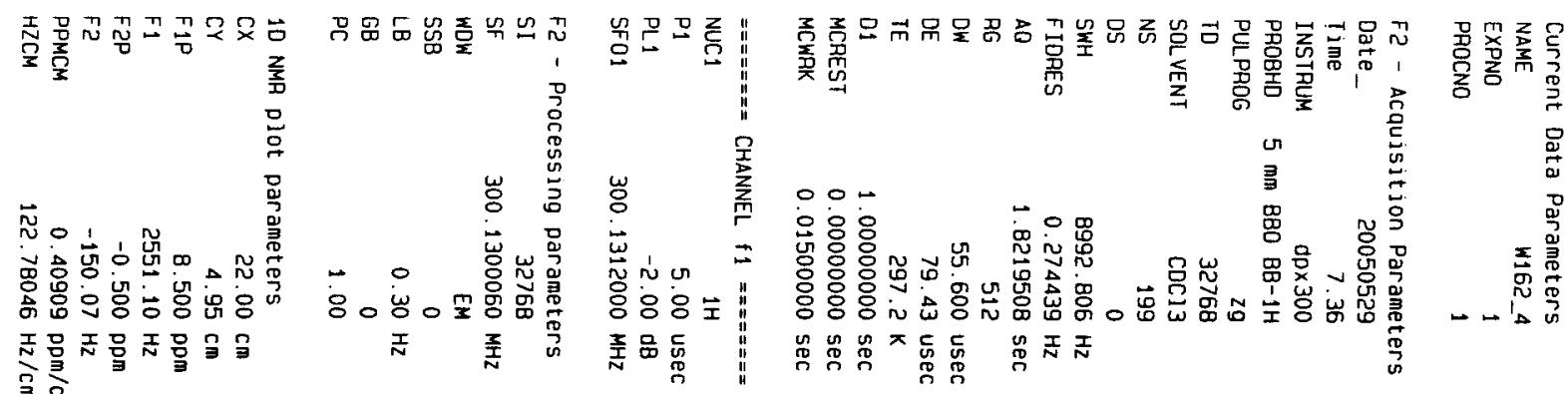




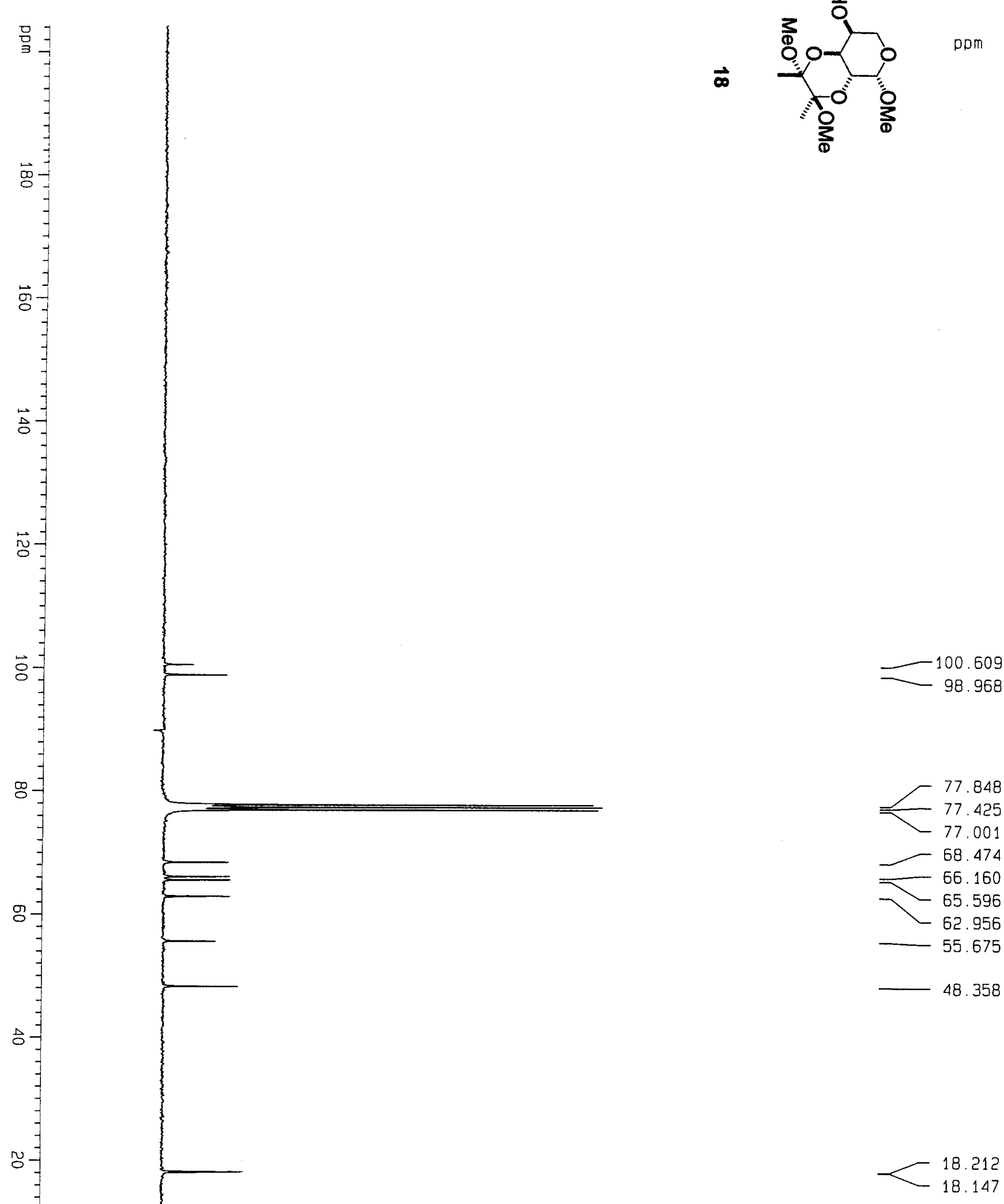



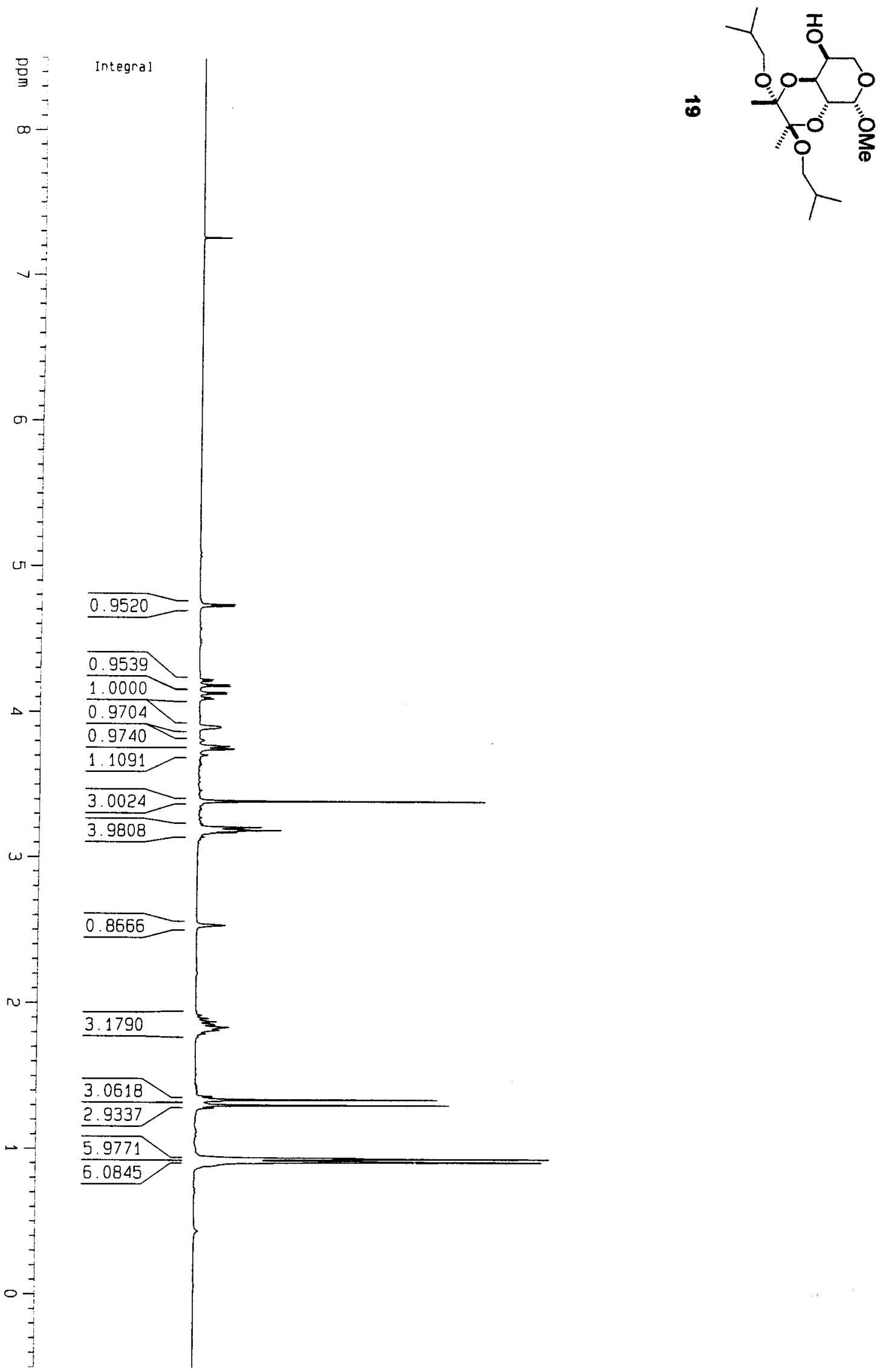

ppm

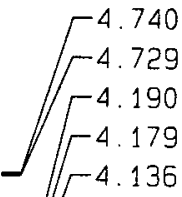

$\Gamma^{4.136}$

$-3.900$

-3.895

$-3.767$

$-3.751$

$-3.746$

$-3.391$

$-3.210$

$-3.202$

$-3.194$

$-3.188$

$-3.182$

-3.172

$-2.534$

$\Gamma^{1.876}$

$-1.859$

$-1.853$

$-1.837$

$-1.815$

$-1.364$

= 1.34

$-1.331$

$-1.302$

$-1.282$

$\leftarrow 0.939$

$-0.932$

$L_{0.916}$

L0.910

$-0.894$

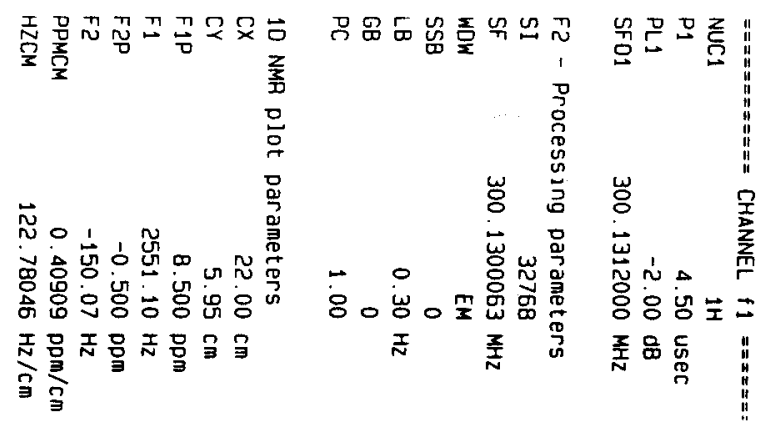

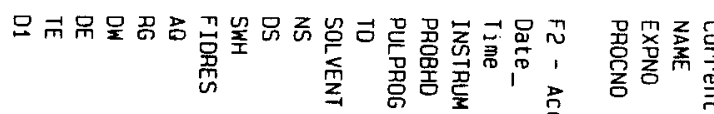

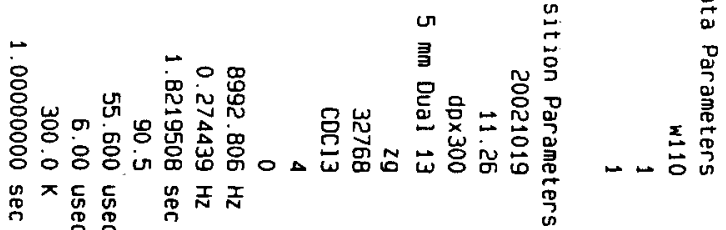



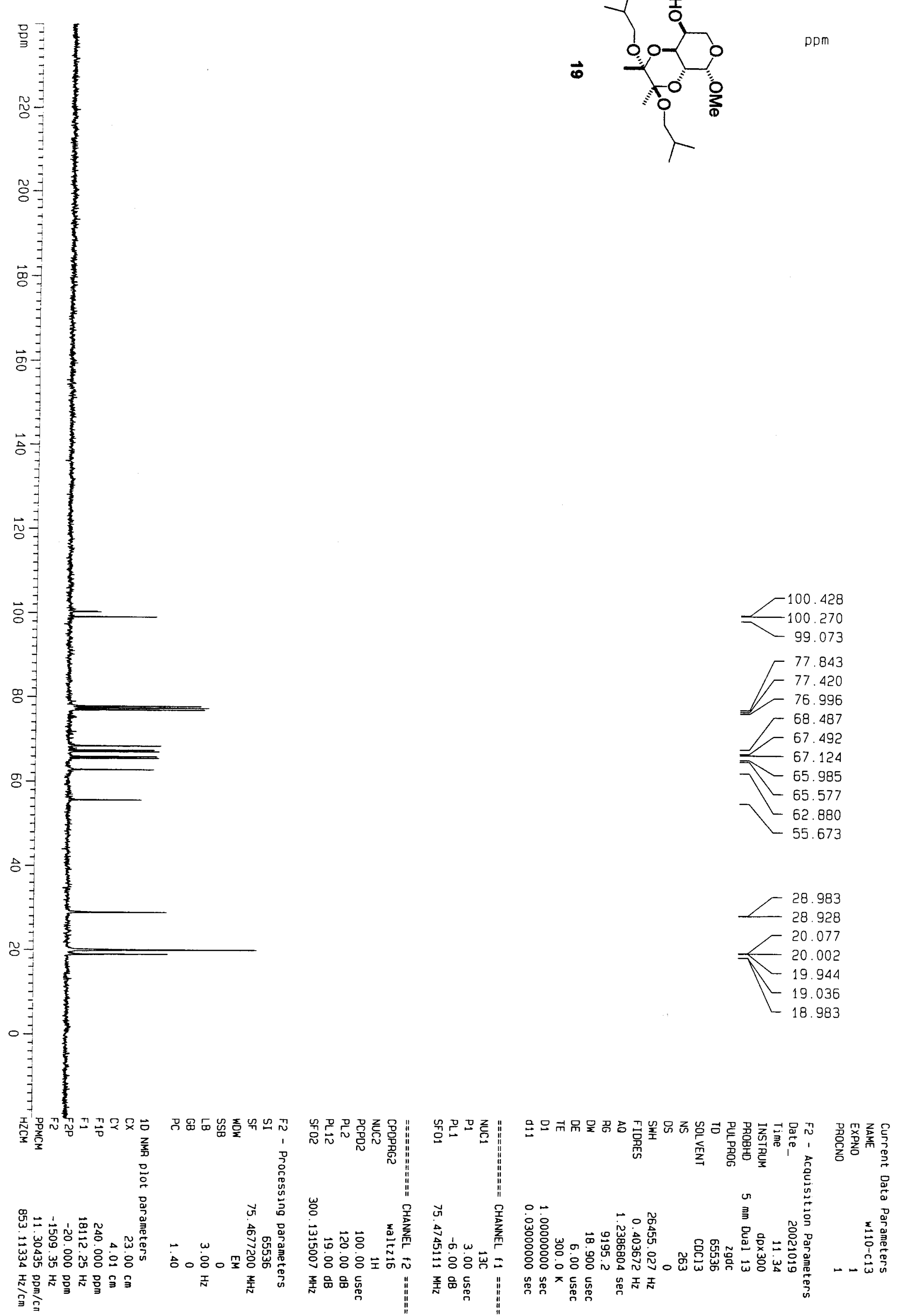


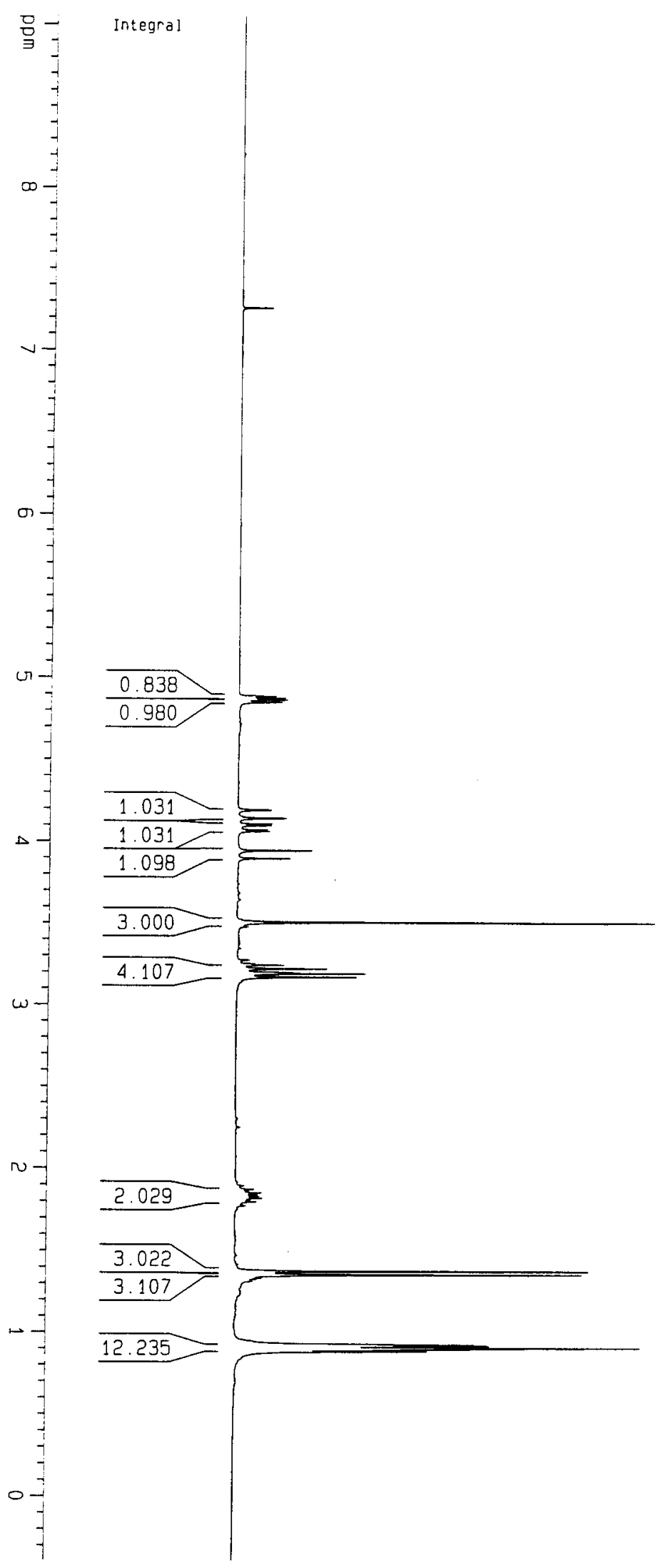

กั

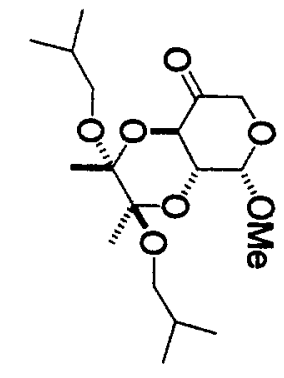

ppm

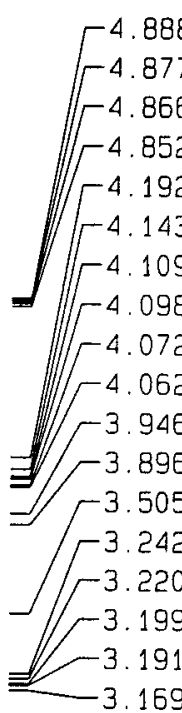

1.874

$-1.852$

$-1.841$

$-1.830$

$\Gamma^{1.819}$

$-1.796$

$\Gamma^{1.376}$

$r^{1.355}$

$-1.335$

$-1.329$

$-1.315$

$-1.240$

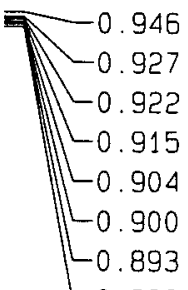

$\leftarrow_{0.882}$

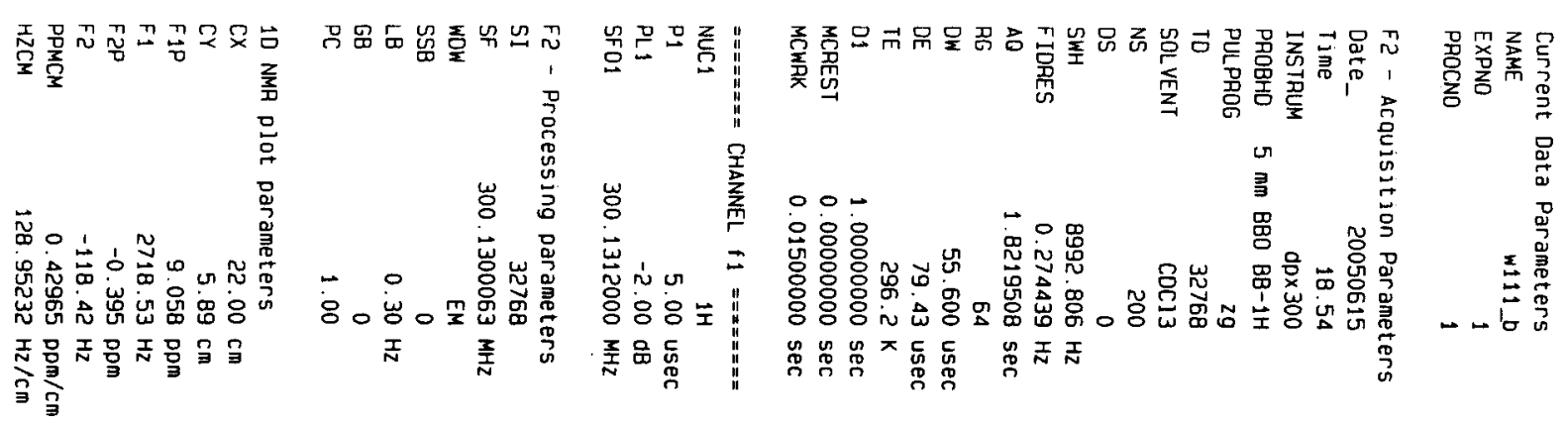



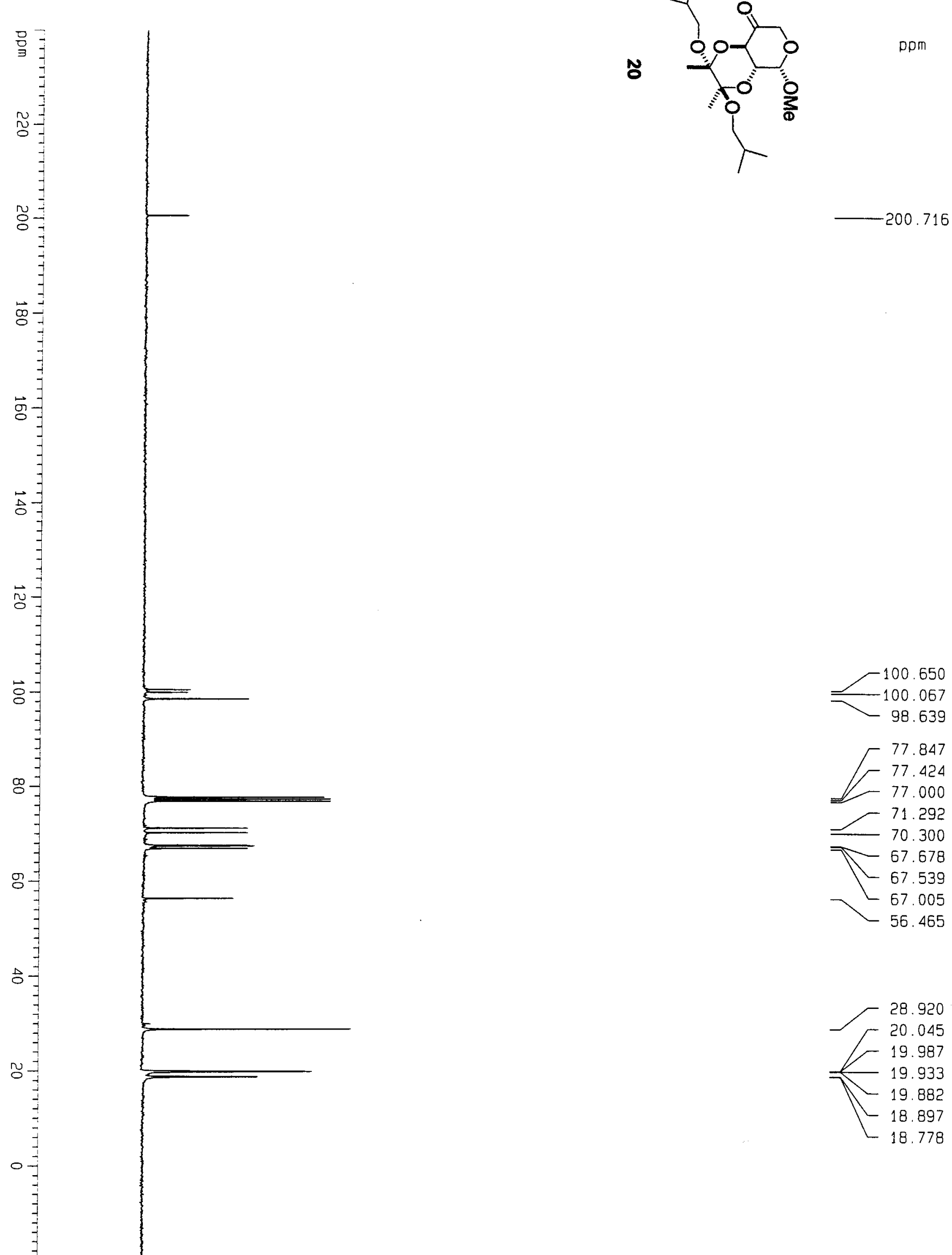

o

g
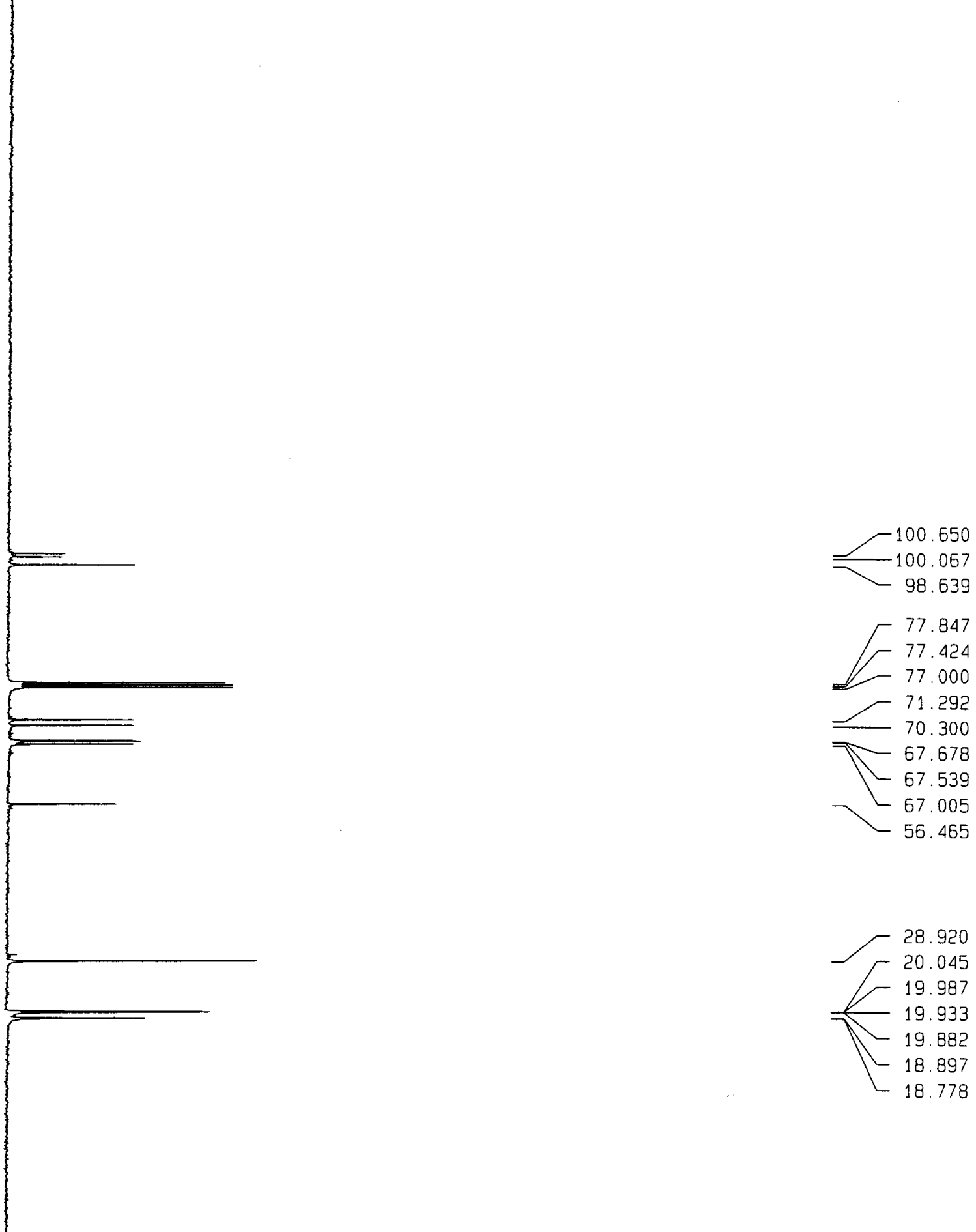

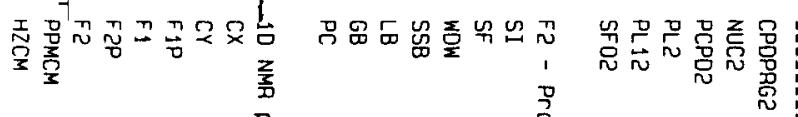

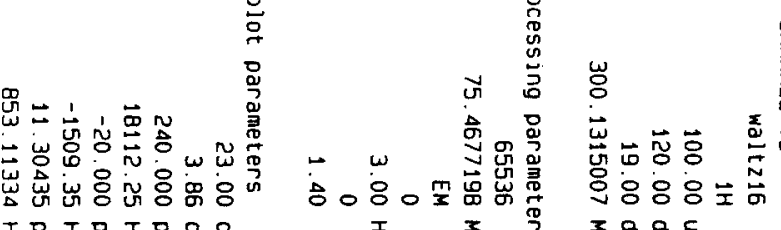

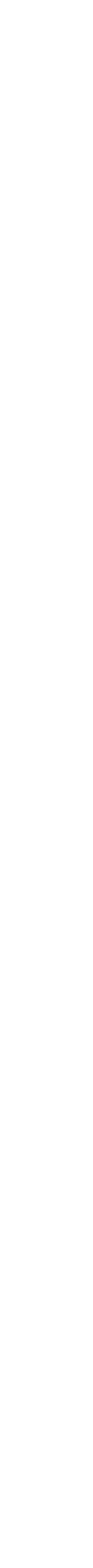

